\title{
Micronutrient intake in advanced age: Te Puāwaitanga o Ngā Tapuwae Kia ora Tonu, Life and Living in Advanced Age: A Cohort Study in New Zealand (LiLACS NZ)
}

\author{
Carol Wham ${ }^{1}$, Ruth Teh ${ }^{2}$, Simon A. Moyes ${ }^{2}$, Anna Rolleston ${ }^{2}$, Marama Muru-Lanning ${ }^{3}$, Karen Hayman ${ }^{2}$, \\ Ngaire Kerse $^{2 *}$ and Ashley Adamson ${ }^{4}$ \\ ${ }^{1}$ School of Food and Nutrition, College of Health, Massey University, North Shore, Auckland 0745, New Zealand \\ ${ }^{2}$ Department of General Practice and Primary Health Care, Faculty of Medical and Health Sciences, University of Auckland, \\ Auckland, Tamaki Campus, Auckland, 1142, New Zealand \\ ${ }^{3}$ James Henare Māori Research Centre, University of Auckland, Auckland, 1142, New Zealand \\ ${ }^{4}$ Human Nutrition Research Centre, Institute of Health E Society and Newcastle University Institute for Ageing, \\ Newcastle University, UK \\ (Submitted 5 December 2015 - Final revision received 31 August 2016 - Accepted 12 September 2016 - First published online 9 November 2016)
}

\section{Abstract}

A high prevalence of undernutrition has previously been reported in indigenous Māori (49\%) and non-Māori (38\%) octogenarians and may be associated with risk of micronutrient deficiencies. We examined vitamin and mineral intakes and the contributing food sources among 216 Māori and 362 non-Māori participating in Life and Living to Advanced age a Cohort Study in New Zealand, using a repeat 24-h multiple-pass recall. More than half of the Mãori and non-Māori participants had intakes below the estimated average requirement from food alone for Ca, Mg and Se. Vitamin $\mathrm{B}_{6}$ (Māori women only), folate (women only), vitamin E (Māori women; all men) and Zn (men only) were low in these ethnic and sex subgroups. Women had intakes of higher nutrient density in folate, vitamin $\mathrm{C}, \mathrm{Ca}, \mathrm{Mg}, \mathrm{K}$, vitamin A (non-Māori) and $\beta$-carotene (Māori) compared with men $(P<0 \cdot 05)$. When controlling for age and physical function, $\beta$-carotene, folate, vitamin $\mathrm{C}$, Ca and Mg were no longer significantly different, but vitamins $\mathrm{B}_{2}, \mathrm{~B}_{12}, \mathrm{E}$ and $\mathrm{D}, \mathrm{Fe}, \mathrm{Na}$, Se and $\mathrm{Zn}$ became significantly different for Māori between men and women. When controlling for age and physical function, vitamins A and $\mathrm{C}$ and Ca were no longer significantly different, but vitamin $\mathrm{B}_{2}$, Fe, Na and $\mathrm{Zn}$ became significantly different for non-Māori between men and women. For those who took nutritional supplements, Māori were less likely to be deficient in food alone intake of vitamin $\mathrm{A}$, folate and $\mathrm{Mg}$, whereas non-Maori were less likely to be deficient in intakes of Mg, $\mathrm{K}$ and $\mathrm{Zn}$, but more likely to be deficient in vitamin $\mathrm{B}_{12}$ intake. A lack of harmonisation in nutrient recommendations hinders the interpretation of nutrient adequacy; nonetheless, $\mathrm{Ca}, \mathrm{Mg}$ and Se are key micronutrients of concern. Milk and cheese were important contributions to Ca intake, whereas bread was a key source of Mg and Se. Examination of dietary intake related to biochemical status and health outcomes will establish the utility of these observations.

\section{Key words: Octogenarians: Dietary intake: Life and Living in Advanced Age: A Cohort Study in New Zealand: Māori}

The number of New Zealanders aged $85+$ years is projected to increase 6-fold by 2051 - an increase from 9 to $22 \%$ of the $65+$ population. Māori are the indigenous people of Aotearoa, New Zealand, comprising $14 \%$ of the total population and $2 \%$ of those aged over 80 years $^{(1)}$. However, the population of Māori aged over 80 years is increasing faster than the non-Māori octogenarian population $^{(2)}$. With the anticipated increase in the population aged 80 years and over, the nutrition-related health of the oldest old, both Māori and non-Māori, is a major public health concern ${ }^{(3)}$.

People in advanced age experience a significant variation in age-related functional changes and have a diverse range of nutritional needs. They also experience a disproportionately high risk of malnutrition and nutrition-related health problems as a result of inadequate food and nutrition intakes ${ }^{(4)}$. In a cohort study of those in advanced age (Te Puāwaitanga o Ngā Tapuwae Kia ora Tonu, Life and Living in Advanced Age: A Cohort Study in New Zealand (LiLACS NZ)), half (49\%) of indigenous Māori and $38 \%$ of non-Māori octogenarians were assessed as being at high nutrition risk using a validated questionnaire - Seniors in the Community: Risk Evaluation for Eating and Nutrition ${ }^{(5)}$.

Micronutrient deficiencies tend to arise because of a reduction in food intake in response to a decline in energy

Abbreviations: AI, adequate intake; EAR, estimated average requirement; EI, energy intake; LiLACS NZ, Life and Living in Advanced Age: A Cohort Study in

New Zealand; NZANS, New Zealand Adult Nutrition Survey; RDI, recommended dietary intake.

* Corresponding author: N. Kerse, fax +6494439640, email n.kerse@auckland.ac.nz 
needs with age. Basal metabolism and energy expenditure for physical activity may be reduced ${ }^{(6)}$, whereas vitamin and mineral needs remain unchanged or are increased $^{(7)}$. Physiological changes may impact the absorption, transport, metabolism and excretion of nutrients ${ }^{(8)}$, and poor health and medications can cause nutrient malabsorption ${ }^{(9,10)}$. Eating habits affected by poor oral health and social isolation may further contribute to lower food intake and nutrient deficiency ${ }^{(11-13)}$. Older people are especially susceptible to vitamin D insufficiency due to reduced mobility, decreased sun exposure and a decline in cutaneous synthesis of vitamin $\mathrm{D}$ with age ${ }^{(14)}$. As micronutrient deficiencies are associated with adverse functional outcomes ${ }^{(15)}$, they may impact the independence of older adults. Therefore, an understanding of micronutrient intakes of those in advanced age is needed.

In New Zealand, there are no nutrients for which the recommended dietary intake (RDI) for older adults aged $71+$ years is less than that for younger adults ${ }^{(16)}$. Of the micronutrients, the RDI for riboflavin and $\mathrm{Ca}$ are higher for adults over 70 years than for younger adults. The adequate intake (AI) for vitamin D (for which there is no RDI) is also higher for this age group.

Data on micronutrient intake in advanced age are limited. Older people in the New Zealand Adult Nutrition Survey (NZANS) are under-represented; data from participants over 70 years and Māori aged over 50 years were aggregated, thus reducing the utility of the data for those in advanced age. This is problematic, as many micronutrient recommendations differ for adults aged over 50 years and beyond ${ }^{(17)}$. Further, older Māori consume different foods according to their cultural preferences, and this may result in different nutrient intakes ${ }^{(18)}$. On the basis of the estimated average requirement (EAR) for men and women aged over 70 years, data from the latest NZANS 2008/09 showed a higher estimated prevalence of low intakes of $\mathrm{Ca}, \mathrm{Zn}$, Se, riboflavin and vitamin $\mathrm{B}_{6}$ compared with younger age groups ${ }^{(19)}$. Multiple micronutrient inadequacies in older people have been reported elsewhere ${ }^{(7,20)}$. A relevant comprehensive analysis to identify micronutrient intake and food sources of micronutrients in people of advanced age is lacking. The aim of this study was to examine energy and micronutrient intakes, and the contribution of food groups towards these intakes, in Māori and non-Māori participating in LiLACS NZ.

\section{Methods}

LiLACS NZ is a population-based cohort study of Māori aged 80-90 years and non-Māori aged 85 years at inception in 2010. Detailed methods have been reported previously ${ }^{(21,22)}$. Maori participants were recruited at a younger age, as the gap in life expectancy between Maori and non-Maori was 8.2 years for men and 8.8 years for women ${ }^{(23)}$. At inception (wave 1 ), the sample consisted of 421 Māori and 516 non-Māori. In brief, participants were identified from the electoral roll, healthcare databases and extensive family and personal networks, and were recruited by personal invitation from the general practitioner or community contact. Those meeting age criteria and living within geographical boundaries of the District Health Boards of the Bay of Plenty and the northern part of the Lakes areas were eligible for participation. The sample recruited was roughly representative of the age structure of the Māori population. Non-Māori women were slightly under-represented compared with the New Zealand population $^{(21)}$.

Wave 2, the 12-month follow-up visit, was completed in 2011 and dietary intake was assessed in 216 Māori and 362 non-Māori octogenarians using two 24-h multiple-pass recalls $(24 \mathrm{~h} \times$ 2 MPR) on two different days. Of the 267 Māori who took part in the 12-month interviews, 216 (81\%) completed the dietary assessment. Māori who completed the dietary assessment did not differ from those who did not with respect to living arrangement, sex, age or depression status. Of the 403 nonMāori who took part in the 12-month interviews, 362 (90\%) completed the dietary assessment; those who completed the dietary assessment did not differ from those who did not complete the assessment with respect to living arrangement, sex, age or depression status.

Supplement use (vitamins, minerals and multivitamins) was recorded by direct observation of pill bottles and recorded in detail by trained interviewers. This study was conducted according to the guidelines laid down in the Declaration of Helsinki, and all procedures involving human subjects were approved by the Northern X Regional Ethics Committee (NXT 09/09/088) in 2009. Written informed consent was obtained from all study participants.

\section{Measures}

In brief, socio-demographic information was collected at Wave 1, baseline interview; living arrangement was categorised as living alone, with spouse only or with others (family members or in residential care). The New Zealand deprivation index was used to estimate socio-economic position ${ }^{(24,25)}$. Weight was measured using the Tanita digital measuring scale (BC-541; Tanita Corporation), and height was measured using a portable stadiometer following established protocols ${ }^{(26)}$. Demispan was used to estimate height for those who were not able to stand ${ }^{(27)}$. BMR was calculated using the Fredrix equation ${ }^{(28)}$. Functional status was assessed using the Nottingham Extended Activities of Daily Living (NEADL) ${ }^{(29)}$, which is a measure of independence in physical function. The NEADL asks whether the older person 'does' a range of activities 'on their own, on their own with difficulty, with help, or not at all'. There are twenty-two items covering four domains: mobility, in the kitchen, domestic tasks and leisure activities. A higher score indicates a higher level of function.

\section{Dietary assessment, nutrient and food group analysis}

At Wave 2, 12-month follow-up, LiLACS NZ participants completed a $24-\mathrm{h} \mathrm{MPR}^{(30)}$ on two separate days of the week conducted by trained interviewers, and FOODfiles (2010), an electronic subset of data from the New Zealand Food Composition Database, was used as the main source of food composition data ${ }^{(31)}$ to calculate mean daily energy and micronutrient intakes. 


\section{Nutrient analysis}

The medians and interquartile ranges of daily energy, vitamin $\mathrm{A}$, $\beta$-carotene, vitamin $\mathrm{B}_{2}, \mathrm{~B}_{6}$, folate, vitamin $\mathrm{B}_{12}, \mathrm{E}, \mathrm{C}$ and $\mathrm{D}, \mathrm{Ca}$, $\mathrm{Fe}, \mathrm{Mg}, \mathrm{K}, \mathrm{Na}$, Se and $\mathrm{Zn}$ intakes were calculated for all participants and repeated after excluding participants with energy intake (EI):BMR $<0.9$ (as potential under-reporters) and $>2.0$ (as potential over-reporters). Nutrient reference values (NRV) for Australia and New Zealand were used to report the proportion of participants meeting the EAR, RDI or AI for the selected nutrients ${ }^{(16)}$. Supplement users were compared with non-supplement users on intake of micronutrients from diet only - that is, not including the contribution from supplements.

\section{Food groups}

Food items reported in the 24-h MPR were allocated to food groups in order to calculate sources of nutrients by the type of food. Recipes were separated into their individual ingredients and these were assigned to separate food groups. The thirtythree food groups used in the 2008/09 NZANS ${ }^{(26)}$ were used to allow national comparisons.

The data analyses were carried out using SAS/STAT software 12.1, version 9.3 of the SAS System for 64-bit Windows 7 Professional. Models were either the univariate non-parametric Mann-Whitney Wilcoxon's test or multivariate generalised linear models controlling for age, sex, physical function (as measured by the NEADL score) and ethnicity as indicated in the text. A $P$ value of $<0.05$ was considered statistically significant.

\section{Results}

Table 1 provides an overview of the social and physical characteristics of the participants and use of dietary supplements. Only $13 \%$ ( $n$ 29) of Māori participants took vitamin supplements, $17 \%$ ( $n$ 37) mineral supplements and 9\% ( $n$ 21) multivitamin and mineral supplements. Nearly a quarter (24\%) of Māori participants took fish oils, glucosamine and herbal supplements. A quarter of non-Māori participants took vitamin (24\%) and mineral (23\%) supplements and 14\% took multivitamin and mineral supplements. Other supplements, for example, glucosamine and herbal supplements, were taken by $37 \%$ of non-Māori participants.

The median daily energy, vitamin and mineral intakes for Māori and non-Māori participants by sex and per MJ of daily total EI are shown in Tables 2 and 3. Although men generally had a higher EI, women had intakes of higher nutrient density (nutrient/MJ) in folate, vitamin $\mathrm{C}, \mathrm{Ca}, \mathrm{Mg}, \mathrm{K}$, vitamin $\mathrm{A}$ (nonMāori) and $\beta$-carotene (Māori) compared with men $(P<0.05)$. The median EI was higher for Māori men than Māori women, whereas intakes per $\mathrm{MJ}$ of $\beta$-carotene, folate, vitamin $\mathrm{C}, \mathrm{Ca}, \mathrm{Mg}$ and $\mathrm{K}$ were higher in Māori women compared with Māori men. When controlling for age and physical function, $\beta$-carotene, folate, vitamin $\mathrm{C}, \mathrm{Ca}$ and $\mathrm{Mg}$ were no longer significantly different but vitamins $\mathrm{B}_{2}, \mathrm{~B}_{12}$, E and $\mathrm{D}, \mathrm{Fe}, \mathrm{Na}$, Se and $\mathrm{Zn}$ became significantly different for Māori (Table 2). Among non-Māori, median energy, vitamin and mineral intakes were higher for men than for women; however, when expressed per MJ energy, intakes of vitamin $\mathrm{A}$, vitamin $\mathrm{B}_{6}$, vitamin $\mathrm{C}, \mathrm{Ca}, \mathrm{Mg}$ and $\mathrm{K}$ were higher in women than in men. When controlling for age and physical function, vitamins $\mathrm{A}, \mathrm{C}$ and $\mathrm{Ca}$ were no longer significantly different, but vitamin $\mathrm{B}_{2}, \mathrm{Fe}, \mathrm{Na}$ and $\mathrm{Zn}$ became significantly different for non-Māori (Table 3).

The daily energy, vitamin and mineral intakes by living situation and level of education for Māori and non-Māori participants are reported in Tables 4 and 5. Māori participants who lived with others (extended family $n 40$ or in residential care $n$ 4) had significantly lower intakes of vitamin A, $\beta$-carotene, vitamin $\mathrm{B}_{6}$, folate, vitamin $\mathrm{C}, \mathrm{Mg}$ and $\mathrm{K}$ compared with those who lived alone or with a spouse (Table 4). Non-Māori participants who lived with others (extended family $n 23$ or in residential care $n$ 18) had significantly lower intakes of $\mathrm{Mg}$ and $\mathrm{K}$ compared with those who lived alone or with a spouse after controlling for age, sex and EI. Non-Māori participants with tertiary education compared with primary or secondary education only had higher intakes of vitamin $\mathrm{B}_{2}$, folate, $\mathrm{Ca}$ and $\mathrm{K}$ after controlling for age, sex and $\mathrm{EI}$ (Table 5).

The percentages of Māori and non-Māori who did not meet the estimated average intake (EAR) or AI for each of the vitamins and minerals included in the analysis (both for those taking supplements or not; intake from food alone reported) are shown in Table 6. Table 6 includes all participants and also presents intakes excluding $35 \%$ of the participants as potential misreporters - that is, reporting EI:BMR $\mathrm{est}<0 \cdot 9$ and $>2 \cdot 0$. Over half of Māori participants had dietary intakes (excluding intake from supplements) of vitamin $\mathrm{B}_{6}, \mathrm{Mg}$, Se and $\mathrm{Zn}$ (men only) below the EAR. Over half did not meet the AI for vitamin E and K. More than $80 \%$ of Māori had intakes below the EAR for $\mathrm{Ca}$, and most of them did not meet the AI for vitamin D (Table 6). Over half of non-Māori participants had intakes of folate (women only), Mg and Se below the EAR, whereas over $80 \%$ had intakes below the EAR for Ca and Zn (men only). For vitamin E, over $40 \%$ of men and women did not meet the AI (Table 6). Women were significantly more likely than men to have folate and vitamin $\mathrm{B}_{12}$ intakes below the EAR, and significantly less likely than men to have vitamin E, Mg, K and Zn intakes below the EAR after controlling for ethnicity. Māori were significantly more likely to have vitamin $\mathrm{B}_{6}$ intakes below the EAR after controlling for sex.

Differences in intake changed after we adjusted to include participants with a EI:BMR between 0.9 and 2.0 only (Table 6), with several of the significant contrasts between Māori and non-Māori becoming non-significant; only vitamin $\mathrm{B}_{6}$ intake remained significantly different between the two ethnic groups. Sensitivity analysis was conducted excluding subjects whose average EI suggested potential misreporting. Those excluded had significantly lower intakes of vitamins $\mathrm{B}_{6}$ and $\mathrm{E}$, $\mathrm{Fe}, \mathrm{Mg}, \mathrm{K}, \mathrm{Na}$ and $\mathrm{Zn}$; although their reported intake was lower for all other micronutrients, it was not significantly so. This is expected as $88 \%$ of Māori excluded and $70 \%$ of non-Māori excluded were deemed to be potentially under-reporting (the difference between Māori and non-Māori was significant, $P=0.03)$. 
Table 1. Social, physical and health characteristics of Mãori and non-Mãori participants by sex (Numbers and percentages; medians and interquartile ranges (IQR))

\begin{tabular}{|c|c|c|c|c|c|c|c|c|c|c|c|c|}
\hline & \multicolumn{6}{|c|}{ Māori } & \multicolumn{6}{|c|}{ Non-Mãori } \\
\hline & \multicolumn{2}{|c|}{ Men } & \multicolumn{2}{|c|}{ Women } & \multicolumn{2}{|c|}{ Total } & \multicolumn{2}{|c|}{ Men } & \multicolumn{2}{|c|}{ Women } & \multicolumn{2}{|c|}{ Total } \\
\hline & $n$ & $\%$ & $n$ & $\%$ & $n$ & $\%$ & $n$ & $\%$ & $n$ & $\%$ & $n$ & $\%$ \\
\hline $\begin{array}{l}\text { Number } \\
\text { Age (years) }\end{array}$ & \multicolumn{2}{|c|}{92} & \multicolumn{2}{|c|}{124} & \multicolumn{2}{|c|}{216} & \multicolumn{2}{|c|}{172} & \multicolumn{2}{|c|}{190} & \multicolumn{2}{|c|}{362} \\
\hline $\begin{array}{l}\text { Median } \\
\text { IQR }\end{array}$ & \multicolumn{2}{|c|}{$\begin{array}{c}82 \\
81,85\end{array}$} & \multicolumn{2}{|c|}{$\begin{array}{c}83.5 \\
81,86\end{array}$} & \multicolumn{2}{|c|}{$\begin{array}{c}83 \\
81,85\end{array}$} & \multicolumn{2}{|c|}{$\begin{array}{c}86 \\
85,86\end{array}$} & \multicolumn{2}{|c|}{$\begin{array}{c}86 \\
85,86\end{array}$} & \multicolumn{2}{|c|}{$\begin{array}{c}86 \\
85,86\end{array}$} \\
\hline \multicolumn{13}{|l|}{ Living arrangement } \\
\hline Alone & 19 & 25 & 54 & 51 & 73 & 40 & 61 & 37 & 120 & 65 & 181 & 52 \\
\hline Spouse only & 35 & 45 & 19 & 18 & 54 & 30 & 96 & 57 & 31 & 17 & 127 & 36 \\
\hline With others* & 23 & 30 & 33 & 31 & 56 & 30 & 10 & 6 & 33 & 18 & 43 & 12 \\
\hline \multicolumn{13}{|l|}{ Deprivation, NZ Dep score } \\
\hline 1-4 least & 12 & 13 & 25 & 20 & 37 & 17 & 46 & 27 & 44 & 23 & 90 & 25 \\
\hline $5-7$ & 26 & 28 & 23 & 19 & 49 & 23 & 73 & 42 & 84 & 44 & 157 & 43 \\
\hline $8-10$ most & 54 & 59 & 76 & 61 & 130 & 60 & 53 & 31 & 62 & 33 & 115 & 32 \\
\hline Smoking & & & & & & & & & & & & \\
\hline Never & 29 & 32 & 63 & 53 & 92 & 43 & 62 & 36 & 130 & 68 & 192 & 53 \\
\hline Current & 10 & 11 & 16 & 13 & 26 & 12 & 11 & 6 & 6 & 3 & 17 & 5 \\
\hline Former & 53 & 58 & 41 & 34 & 94 & 44 & 99 & 58 & 54 & 28 & 153 & 42 \\
\hline Alcohol & & & & & & & & & & & & \\
\hline Never & 32 & 42 & 51 & 48 & 83 & 46 & 31 & 18 & 73 & 40 & 104 & 29 \\
\hline Monthly or less & 12 & 16 & 22 & 21 & 34 & 19 & 23 & 14 & 41 & 22 & 64 & 18 \\
\hline 2-4 times a month & 7 & 9 & 10 & 9 & 17 & 9 & 19 & 11 & 20 & 11 & 39 & 11 \\
\hline 2-3times a week & 7 & 9 & 7 & 7 & 14 & 8 & 20 & 12 & 18 & 10 & 38 & 11 \\
\hline$\geq 4$ times a week & 18 & 24 & 16 & 15 & 34 & 19 & 76 & 45 & 32 & 17 & 108 & 31 \\
\hline Dietary supplement usage & & & & & & & & & & & & \\
\hline Vitamins & 10 & 11 & 19 & 15 & 29 & 13 & 26 & 15 & 62 & 31 & 88 & 24 \\
\hline Minerals & 14 & 15 & 23 & 18 & 37 & 17 & 31 & 18 & 52 & 26 & 83 & 23 \\
\hline Multivitamins and minerals & 6 & 6 & 15 & 12 & 21 & 9 & 19 & 11 & 32 & 16 & 51 & 14 \\
\hline Other† & 19 & 20 & 34 & 26 & 53 & 24 & 51 & 30 & 83 & 42 & 134 & 37 \\
\hline Physical function NEADL & $15 \cdot 4$ & $4 \cdot 8$ & $16 \cdot 9$ & 4.5 & $16 \cdot 3$ & $4 \cdot 7$ & $16 \cdot 5$ & 3.9 & $16 \cdot 9$ & $4 \cdot 8$ & $16 \cdot 7$ & $4 \cdot 4$ \\
\hline Weight (kg) & & & & & & & & & & & & \\
\hline Median & & & & & & & & & & & & \\
\hline IQR & & 35.4 & & 7.9 & $59 \cdot 8$ & & & & $57 \cdot 3$ & & $60 \cdot \varepsilon$ & \\
\hline BMI $\left(\mathrm{kg} / \mathrm{m}^{2}\right)$ & & & & & & & & & & & & \\
\hline Median & & & & & & & & & & & & \\
\hline IQR & & $31 \cdot 1$ & $24 .($ & 1.6 & 24.7 & & & 8.5 & 23.7 & 0.0 & 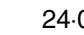 & 29.2 \\
\hline BMR (kJ/d) & & & & & & & & & & & & \\
\hline Median & & & & & & & & & & & & \\
\hline IQR & 615 & 7255 & 489 & 904 & 5109 & 686 & 614 & 004 & 4690 & 334 & 4975 & 565 \\
\hline BMR (kcal/d) & & & & & & & & & & & & \\
\hline Median & & & & & & & & & & & & \\
\hline IQR & 147 & 1734 & 117 & 411 & 1221 & 598 & 146 & 674 & 1121 & 275 & $118 s$ & 1569 \\
\hline
\end{tabular}

NEADL, Nottingham Extended Activities of Daily Living score ${ }^{(29)}$

* With others includes living with extended family, and eight participants in residential care.

† Fish oils, glucosamine and herbal.

Māori who took supplements (vitamins, multivitamins or minerals) were less likely to have a food intake deficient in vitamin A, folate and $\mathrm{Mg}$ (Table 7). Non-Maori who took supplements (vitamins, multivitamins or minerals) were less likely to have a diet deficient in $\mathrm{Mg}, \mathrm{K}$ and $\mathrm{Zn}$ but were more likely to have a diet deficient in vitamin $\mathrm{B}_{12}$.

The food groups that contribute to $\geq 75 \%$ of vitamin and mineral intakes for Māori and non-Māori men and women are presented in Fig. 1. Fig. 2 and 3 show the distribution of intakes for Se and folate for Māori and non-Māori by sex with the EAR and recommended daily intake (RDI) indicated. Se and folate are known to be of particular concern in this population, and over half of both Māori and non-Māori had intakes below the EAR (except folate for non-Māori men). Se is known to be low in New Zealand soil, and older people are especially vulnerable to folate deficiency ${ }^{(32)}$. Sex differences in food group sources of folate were found.

\section{Food sources: Māori participants}

As shown in Fig. 1, vegetables provided the largest percentage of food group contribution of vitamin A ( $42 \%$ for women and $35 \%$ for men) followed by butter and margarine (approximately $11 \%$ ). Cereals (approximately 19\%), bread (approximately $19 \%$ ) and vegetables (approximately 15\%) were the three main food sources for folate intake. The main food sources of vitamin $\mathrm{B}_{12}$ were milk, fish and seafood, and beef and veal. These three 
Table 2. Daily energy, vitamin and mineral intakes from food for Mãori participants by sex and per MJ of energy (Medians and interquartile ranges (IQR))

\begin{tabular}{|c|c|c|c|c|c|c|c|c|c|c|}
\hline \multirow[b]{2}{*}{ Micronutrients } & \multicolumn{2}{|c|}{ All } & \multicolumn{3}{|c|}{ Men } & \multicolumn{3}{|c|}{ Women } & \multirow[b]{2}{*}{$P^{*}$} & \multirow[b]{2}{*}{$P \dagger$} \\
\hline & Median & IQR & Median & IQR & Per $1 \mathrm{MJ}$ & Median & IQR & Per $1 \mathrm{MJ}$ & & \\
\hline Energy (MJ) & $6 \cdot 4$ & $5 \cdot 2-8 \cdot 2$ & 7.4 & $6 \cdot 1-9 \cdot 1$ & - & $6 \cdot 0$ & $4 \cdot 8-7 \cdot 2$ & - & $<0.0001$ & $<0.0001$ \\
\hline \multicolumn{11}{|l|}{ Vitamins } \\
\hline Vitamin A ( $\mu \mathrm{g} R E)$ & 859 & $549-1278$ & 1020 & $559-1405$ & $123 \cdot 0$ & 829 & $541-1169$ & $136 \cdot 0$ & 0.143 & 0.133 \\
\hline$\beta$-Carotene $(\mu \mathrm{g})$ & 3011 & $1228-4946$ & 2805 & $1014-5310$ & 354.5 & 3102 & $1310-4923$ & 453.8 & 0.023 & 0.399 \\
\hline Vitamin $B_{2}(\mathrm{mg})$ & 1.6 & $1 \cdot 1-2 \cdot 0$ & 1.7 & $1 \cdot 2-2 \cdot 3$ & 0.2 & 1.5 & $1 \cdot 1-2 \cdot 0$ & 0.2 & 0.346 & 0.003 \\
\hline Vitamin $B_{6}(\mathrm{mg})$ & $1 \cdot 2$ & $0.9-1.7$ & 1.3 & $1 \cdot 0-1 \cdot 8$ & 0.2 & $1 \cdot 2$ & $0.9-1.7$ & 0.2 & 0.187 & 0.065 \\
\hline Folate $(\mu \mathrm{g})$ & 199 & $156-262$ & 206 & $160-271$ & 28.5 & 193 & $151-252$ & $32 \cdot 2$ & 0.002 & 0.076 \\
\hline Vitamin $B_{12}(\mu \mathrm{g})$ & 3.0 & $1.9-4.4$ & 3.4 & $2 \cdot 5-5 \cdot 1$ & 0.5 & 2.7 & $1.7-3.9$ & 0.5 & 0.199 & 0.027 \\
\hline Vitamin E (mg) & $7 \cdot 0$ & $4 \cdot 8-9 \cdot 4$ & $7 \cdot 7$ & $5 \cdot 4-10 \cdot 6$ & 1.0 & 6.4 & $4.6-8.9$ & 1.0 & 0.088 & 0.021 \\
\hline Vitamin C (mg) & 71.6 & $46 \cdot 1-124 \cdot 3$ & 74.5 & $43 \cdot 3-120 \cdot 5$ & $10 \cdot 7$ & $70 \cdot 6$ & $47 \cdot 1-126 \cdot 5$ & $12 \cdot 8$ & 0.016 & 0.734 \\
\hline Vitamin D ( $\mu \mathrm{g})$ & 3.2 & $1 \cdot 8-5 \cdot 7$ & 3.8 & $2 \cdot 4-7 \cdot 2$ & 0.5 & 3.0 & $1.7-4.5$ & 0.5 & 0.443 & 0.005 \\
\hline \multicolumn{11}{|l|}{ Minerals } \\
\hline $\mathrm{Ca}(\mathrm{mg})$ & 563 & $424-778$ & 578 & $414-859$ & $76 \cdot 2$ & 543 & $433-765$ & $94 \cdot 1$ & 0.009 & 0.139 \\
\hline $\mathrm{Fe}(\mathrm{mg})$ & 9.7 & $7 \cdot 1-13 \cdot 1$ & $11 \cdot 2$ & $7 \cdot 8-14 \cdot 4$ & 1.4 & 8.9 & $6.7-11.9$ & 1.5 & 0.377 & 0.001 \\
\hline $\mathrm{Mg}(\mathrm{mg})$ & 217 & 179-272 & 235 & $188-286$ & $32 \cdot 1$ & 208 & $162-258$ & 35.5 & 0.004 & 0.053 \\
\hline $\mathrm{K}(\mathrm{mg})$ & 2374 & $1883-3006$ & 2624 & 2055-3332 & 334.3 & 2250 & $1809-2805$ & 379.5 & 0.004 & 0.0004 \\
\hline $\mathrm{Na}(\mathrm{mg}) \ddagger$ & 2305 & $1760-3124$ & 2720 & $1809-3351$ & 348.7 & 2169 & $1660-2770$ & $364 \cdot 2$ & 0.281 & 0.0003 \\
\hline $\mathrm{Se}(\mu \mathrm{g})$ & 37.8 & $25 \cdot 2-59 \cdot 8$ & $44 \cdot 3$ & $31 \cdot 8-66 \cdot 6$ & $6 \cdot 3$ & $32 \cdot 4$ & $22 \cdot 4-57.0$ & 5.8 & 0.577 & 0.016 \\
\hline $\mathrm{Zn}(\mathrm{mg})$ & $7 \cdot 7$ & $5.8-10.5$ & 8.9 & $6 \cdot 8-13$ & 1.2 & $7 \cdot 1$ & $5 \cdot 3-9 \cdot 1$ & 1.2 & 0.778 & 0.0001 \\
\hline
\end{tabular}

$\mathrm{RE}$, retinol equivalents.

* Mann-Whitney $U$ test for no sex difference (per $1 \mathrm{MJ}$ of energy).

† Comparing all men and all women, multivariate generalised linear model controlling for age and functional status from the Nottingham Extended Activities of Daily Living Scale score ${ }^{(29)}$.

‡ Does not include intake from supplements, energy from alcohol, table salt and salt used for cooking. 
Table 3. Daily energy, vitamin and mineral intakes from food for non-Mãori participants by sex and per MJ of energy (Medians and interquartile ranges (IQR))

\begin{tabular}{|c|c|c|c|c|c|c|c|c|c|c|}
\hline \multirow[b]{2}{*}{ Micronutrients } & \multicolumn{2}{|c|}{ All } & \multicolumn{3}{|c|}{ Men } & \multicolumn{3}{|c|}{ Women } & \multirow[b]{2}{*}{$P^{*}$} & \multirow[b]{2}{*}{$P \dagger$} \\
\hline & Median & IQR & Median & IQR & Per $1 \mathrm{MJ}$ & Median & IQR & Per $1 \mathrm{MJ}$ & & \\
\hline Energy (MJ) & $7 \cdot 0$ & $5 \cdot 8-8 \cdot 7$ & 7.9 & $6 \cdot 7-9 \cdot 6$ & - & $6 \cdot 3$ & $5 \cdot 3-7 \cdot 5$ & - & $<0.0001$ & $<0.0001$ \\
\hline \multicolumn{11}{|l|}{ Vitamins } \\
\hline Vitamin A ( $\mu \mathrm{g} R E)$ & 904 & 628-1278 & 981 & $672-1323$ & 115.9 & 867 & $571-1230$ & 141.6 & 0.01 & 0.606 \\
\hline$\beta$-Carotene $(\mu \mathrm{g})$ & 3029 & $1823-4937$ & 3325 & $1960-5163$ & $406 \cdot 6$ & 2831 & $1809-4485$ & 431.4 & 0.15 & 0.195 \\
\hline Vitamin $B_{2}(\mathrm{mg})$ & $1 \cdot 8$ & $1 \cdot 4-2 \cdot 3$ & $2 \cdot 0$ & $1.5-2.5$ & 0.2 & 1.6 & $1 \cdot 2-2 \cdot 2$ & 0.3 & 0.062 & $<0.0001$ \\
\hline Vitamin $B_{6}(\mathrm{mg})$ & 1.4 & $1 \cdot 1-1.9$ & 1.5 & $1 \cdot 2-2 \cdot 1$ & 0.2 & 1.4 & $1 \cdot 1-1 \cdot 7$ & 0.2 & 0.001 & 0.012 \\
\hline Folate $(\mu \mathrm{g})$ & 233 & $178-298$ & 245 & $192-316$ & $30 \cdot 2$ & 215 & 163-285 & 33.9 & 0.002 & 0.0001 \\
\hline Vitamin $B_{12}(\mu \mathrm{g})$ & $3 \cdot 0$ & $2 \cdot 1-4 \cdot 2$ & 3.6 & $2 \cdot 4-4 \cdot 8$ & 0.5 & $2 \cdot 6$ & $1 \cdot 8-3 \cdot 6$ & 0.4 & 0.37 & $0 \cdot 162$ \\
\hline Vitamin E (mg) & 8.4 & $6 \cdot 2-11.5$ & 9.5 & $7 \cdot 2-11 \cdot 8$ & $1 \cdot 1$ & $7 \cdot 6$ & $5 \cdot 5-10.5$ & 1.2 & 0.054 & 0.215 \\
\hline Vitamin C (mg) & 85.4 & $54 \cdot 2-135 \cdot 8$ & $85 \cdot 6$ & $57 \cdot 5-136 \cdot 6$ & 10.5 & 84.5 & $51 \cdot 2-133 \cdot 8$ & $13 \cdot 2$ & 0.002 & 0.881 \\
\hline Vitamin D $(\mu \mathrm{g})$ & 3.7 & $2 \cdot 3-5 \cdot 9$ & $4 \cdot 1$ & $2 \cdot 8-6 \cdot 3$ & 0.5 & 3.4 & $2 \cdot 0-5 \cdot 5$ & 0.5 & 0.613 & 0.086 \\
\hline \multicolumn{11}{|l|}{ Minerals } \\
\hline $\mathrm{Ca}(\mathrm{mg})$ & 702 & $541-905$ & 731 & $582-928$ & 88.8 & 679 & $507-852$ & $109 \cdot 2$ & $<0.0001$ & 0.193 \\
\hline $\mathrm{Fe}(\mathrm{mg})$ & $10 \cdot 6$ & $8 \cdot 1-13 \cdot 3$ & 11.6 & $9 \cdot 9-14 \cdot 3$ & 1.4 & $9 \cdot 3$ & $7 \cdot 1-11 \cdot 7$ & 1.4 & 0.693 & $<0.0001$ \\
\hline $\mathrm{Mg}(\mathrm{mg})$ & 258 & $214-321$ & 276 & 229-331 & 33.9 & 244 & 190-309 & 38.6 & $<0.0001$ & 0.004 \\
\hline $\mathrm{K}(\mathrm{mg})$ & 2755 & 2243-3285 & 2994 & $2544-3450$ & $361 \cdot 8$ & 2508 & 2118-3087 & $400 \cdot 2$ & 0.0001 & $<0.0001$ \\
\hline $\mathrm{Na}(\mathrm{mg}) \ddagger$ & 2485 & $1869-3147$ & 2764 & 2278-3377 & 348.1 & 2189 & $1658-2823$ & $342 \cdot 3$ & 0.996 & $<0.0001$ \\
\hline Se $(\mu \mathrm{g})$ & 39.5 & $27 \cdot 0-56 \cdot 5$ & $45 \cdot 8$ & $32 \cdot 6-61 \cdot 2$ & $5 \cdot 3$ & 34.0 & $24 \cdot 6-47 \cdot 6$ & $5 \cdot 1$ & 0.345 & 0.232 \\
\hline $\mathrm{Zn}(\mathrm{mg})$ & 8.4 & $6.5-10.3$ & 9.4 & $7 \cdot 6-11 \cdot 2$ & 1.2 & 7.4 & $5 \cdot 8-9 \cdot 3$ & 1.2 & 0.43 & $<0.0001$ \\
\hline
\end{tabular}

$\mathrm{RE}$, retinol equivalents.

* Mann-Whitney $U$ test for no sex difference (per 1 MJ of energy).

† Comparing all men and all women, multivariate generalised linear model controlling for physical functional status from the Nottingham Extended Activities of Daily Living Scale score ${ }^{(29)}$.

‡ Does not include intake from supplements, energy from alcohol, table salt and salt used for cooking. 
Table 4. Daily energy, vitamin and mineral intake from food for Mãori participants by living situation and education

\begin{tabular}{|c|c|c|c|c|c|c|c|}
\hline \multirow[b]{2}{*}{ Micronutrients } & \multicolumn{3}{|c|}{ Living situation } & \multicolumn{4}{|c|}{ Education } \\
\hline & $\begin{array}{l}\text { Alone } \\
(n 73)\end{array}$ & $\begin{array}{l}\text { Spouse only } \\
\text { (n 54) }\end{array}$ & $\begin{array}{l}\text { With Others } \\
\quad(n 56)\end{array}$ & $\begin{array}{l}\text { Primary only } \\
\quad(n 65)\end{array}$ & $\begin{array}{l}\text { Secondary } \\
\quad(n 123)\end{array}$ & $\begin{array}{l}\text { Trade qualification } \\
\qquad(n 7)\end{array}$ & $\begin{array}{l}\text { Tertiary } \\
(n 16)\end{array}$ \\
\hline Energy (MJ) & $6 \cdot 3$ & $7 \cdot 1$ & 6.4 & $6 \cdot 7$ & $6 \cdot 3$ & $6 \cdot 3$ & $6 \cdot 1$ \\
\hline \multicolumn{8}{|l|}{ Vitamins } \\
\hline Vitamin A ( $\mu \mathrm{g} R E)$ & 954 & 1141 & $760^{\star}$ & 1046 & 764 & 954 & 937 \\
\hline$\beta$-Carotene $(\mu \mathrm{g})$ & 3292 & 3849 & $2344^{\star \star}$ & 3137 & 2661 & 4823 & 3826 \\
\hline Vitamin $B_{2}(\mathrm{mg})$ & 1.5 & 1.7 & 1.6 & 1.6 & 1.6 & 1.6 & 1.7 \\
\hline Vitamin $\mathrm{B}_{6}(\mathrm{mg})$ & 1.4 & 1.5 & $1 \cdot 1^{\star *}$ & 1.2 & 1.2 & 1.5 & 1.2 \\
\hline Folate $(\mu \mathrm{g})$ & 216 & 232 & $191^{*}$ & 189 & 206 & 186 & 187 \\
\hline Vitamin $\mathrm{B}_{12}(\mu \mathrm{g})$ & 3.2 & $3 \cdot 2$ & 2.9 & 2.9 & 3.0 & 1.9 & 3.3 \\
\hline Vitamin E (mg) & 7.6 & 7.8 & $6 \cdot 1$ & $7 \cdot 2$ & 6.6 & $7 \cdot 1$ & $6 \cdot 7$ \\
\hline Vitamin C (mg) & $76 \cdot 3$ & 96.5 & $65 \cdot 1^{\star \star}$ & $68 \cdot 2$ & $72 \cdot 0$ & 67.4 & 94.0 \\
\hline Vitamin $D(\mu \mathrm{g})$ & 2.9 & 3.3 & 3.3 & 3.3 & 2.9 & 3.5 & 3.3 \\
\hline \multicolumn{8}{|l|}{ Minerals } \\
\hline $\mathrm{Ca}(\mathrm{mg})$ & 546 & 633 & 582 & 543 & 565 & 586 & 744 \\
\hline $\mathrm{Fe}(\mathrm{mg})$ & 9.5 & 11.0 & 9.5 & 10.7 & 9.5 & 9.2 & $10 \cdot 1$ \\
\hline $\mathrm{Mg}(\mathrm{mg})$ & 217 & 260 & $202^{\star \star}$ & 203 & 224 & 259 & 209 \\
\hline $\mathrm{K}(\mathrm{mg})$ & 2370 & 2806 & $2304^{* *}$ & 2370 & 2367 & 2752 & 2637 \\
\hline $\mathrm{Na}(\mathrm{mg}) \dagger$ & 2315 & 2513 & 2271 & 2290 & 2304 & 2370 & 2304 \\
\hline Se $(\mu \mathrm{g})$ & 34.7 & 41.3 & 41.6 & 43.7 & 34.7 & 42.9 & 35.5 \\
\hline $\mathrm{Zn}(\mathrm{mg})$ & $8 \cdot 1$ & 8.4 & $7 \cdot 2$ & $7 \cdot 8$ & 7.5 & 8.9 & 7.4 \\
\hline
\end{tabular}

${ }^{*} P<0.05,{ }^{* *} P<0.01$, comparing all living situations or all education levels, multivariate generalised linear model controlling for age, sex and energy intake.

$\dagger$ Does not include intake from supplements, table salt and salt for cooking.

Table 5. Daily energy, vitamin and mineral intakes from food for non-Māori participants by living situation and education

\begin{tabular}{|c|c|c|c|c|c|c|c|}
\hline \multirow[b]{2}{*}{ Micronutrients } & \multicolumn{3}{|c|}{ Live with } & \multicolumn{4}{|c|}{ Education } \\
\hline & $\begin{array}{l}\text { Alone } \\
(n \text { 181) }\end{array}$ & $\begin{array}{l}\text { Spouse only } \\
(n 127)\end{array}$ & $\begin{array}{l}\text { With Others } \\
\quad(n \text { 43) }\end{array}$ & $\begin{array}{l}\text { Primary only } \\
\quad(n \text { 63) }\end{array}$ & $\begin{array}{l}\text { Secondary } \\
\text { (n 199) }\end{array}$ & $\begin{array}{l}\text { Trade qualification } \\
\qquad(n \text { 42) }\end{array}$ & $\begin{array}{l}\text { Tertiary } \\
\text { ( } n \text { 55) }\end{array}$ \\
\hline Energy (MJ) & $6 \cdot 7$ & 7.7 & $6 \cdot 6$ & $7 \cdot 1$ & 6.9 & $6 \cdot 7$ & 7.7 \\
\hline \multicolumn{8}{|l|}{ Vitamins } \\
\hline Vitamin A ( $\mu \mathrm{g} R E)$ & 904 & 920 & 776 & 869 & 919 & 884 & 1076 \\
\hline$\beta$-Carotene $(\mu \mathrm{g})$ & 3291 & 2888 & 2816 & 2328 & 3247 & 2676 & 3522 \\
\hline Vitamin $B_{2}(\mathrm{mg})$ & 1.7 & 1.9 & 1.6 & 1.8 & 1.7 & 1.7 & $2 \cdot 3^{*}$ \\
\hline Vitamin $B_{6}(\mathrm{mg})$ & 1.5 & 1.4 & $1 \cdot 2$ & 1.3 & 1.4 & 1.4 & 1.6 \\
\hline Folate $(\mu \mathrm{g})$ & 237 & 240 & 204 & 222 & 229 & 238 & $272^{\star}$ \\
\hline Vitamin $B_{12}(\mu \mathrm{g})$ & $2 \cdot 8$ & 3.6 & 2.5 & $2 \cdot 8$ & $2 \cdot 9$ & $3 \cdot 2$ & 3.7 \\
\hline Vitamin E (mg) & 7.9 & 9.7 & 7.4 & 8.0 & 8.5 & 8.3 & 9.9 \\
\hline Vitamin C (mg) & $92 \cdot 6$ & 87.4 & 75.9 & $76 \cdot 8$ & 85.5 & $80 \cdot 2$ & 109.7 \\
\hline Vitamin D $(\mu \mathrm{g})$ & 3.5 & $4 \cdot 2$ & 3.7 & 3.9 & 3.5 & 3.6 & 3.9 \\
\hline \multicolumn{8}{|l|}{ Minerals } \\
\hline $\mathrm{Ca}(\mathrm{mg})$ & 705 & 720 & 701 & 757 & 693 & 635 & $809^{\star}$ \\
\hline $\mathrm{Fe}(\mathrm{mg})$ & $10 \cdot 2$ & $11 \cdot 1$ & 9.6 & $10 \cdot 3$ & 10.5 & $10 \cdot 3$ & 11.3 \\
\hline $\mathrm{Mg}(\mathrm{mg})$ & 261 & 260 & $215^{\star \star}$ & 254 & 255 & 262 & 290 \\
\hline $\mathrm{K}(\mathrm{mg})$ & 2761 & 2830 & $2342^{\star \star \star}$ & 2633 & 2724 & 2601 & $3133^{*}$ \\
\hline $\mathrm{Na}(\mathrm{mg}) \dagger$ & 2344 & 2739 & 2510 & 2373 & 2544 & 2214 & 2721 \\
\hline Se $(\mu \mathrm{g})$ & $39 \cdot 2$ & $41 \cdot 1$ & 37.9 & $39 \cdot 1$ & 39.9 & $38 \cdot 2$ & $42 \cdot 4$ \\
\hline $\mathrm{Zn}(\mathrm{mg})$ & $8 \cdot 1$ & 8.9 & 6.5 & $8 \cdot 3$ & $8 \cdot 1$ & 8.5 & 9.4 \\
\hline
\end{tabular}

${ }^{\star} P<0.05,{ }^{\star \star} P<0.01,{ }^{\star \star \star} P<0.001$ comparing all living situations or all education levels, multivariate generalised linear model controlling for age, sex and energy intake. $\dagger$ Does not include intake from supplements, table salt and salt for cooking.

food groups contributed to more than half of the vitamin $\mathrm{B}_{12}$ intake among Māori men (52\%) and Māori women (54\%). The three main food group contributors of vitamin D were milk (approximately 26\%), butter and margarine (approximately $10 \%$ ), and fish and seafood (approximately 14\%). Milk was the largest contributor to dietary $\mathrm{Ca}$ intake for men (33\%) and women (34\%) followed by bread for Māori women (10\%). Cereals contributed about $17 \%$ to dietary Fe intake followed by bread (men $12 \%$, women 15\%), vegetables (men $7 \%$, women
$8 \%$ ), beef and veal ( $8 \%$ for both men and women), and nonalcoholic beverages ( $5 \%$ for both men and women). For Māori women, the main food groups for Mg were bread (16\%), fruits (10\%) and cereals (10\%); for Māori men, the three main sources were bread (14\%), cereals (11\%), and potatoes, kumara and taro (9\%). The main food group contributing to $\mathrm{K}$ intake in men was potatoes, kumara and taro (>16\%); for women this was fruit (14\%), followed closely by vegetables (13\%). Fish and seafood (about $21 \%$ ) and bread (12\% for men and 15\% for 
Table 6. Proportion of all Māori and non-Māori participants who did not meet the nutrient reference values (NRV) and, for participants with an energy intake (El):BMR of between 0.9 and 2.0, who did not meet the NRV for Australia and New Zealand ${ }^{(16)}$ for daily intake of micronutrients

(Numbers and percentages)

\begin{tabular}{|c|c|c|c|c|c|c|c|c|c|c|c|c|}
\hline \multirow[b]{3}{*}{ Micronutrients } & \multicolumn{4}{|c|}{ Women } & \multirow[b]{3}{*}{ Women $>70$ years NRV } & \multicolumn{4}{|c|}{ Men } & \multirow[b]{3}{*}{ Men $>70$ years NRV } & \multirow[b]{3}{*}{$P_{\text {sex }}$} & \multirow[b]{3}{*}{$P_{\text {ethnic group }}$} \\
\hline & \multicolumn{2}{|c|}{ Māori } & \multicolumn{2}{|c|}{ Non-Māori } & & \multicolumn{2}{|c|}{ Mãori } & \multicolumn{2}{|c|}{ Non-Māori } & & & \\
\hline & $n$ & $\%$ & $n$ & $\%$ & & $n$ & $\%$ & $n$ & $\%$ & & & \\
\hline \multicolumn{13}{|l|}{ All participants } \\
\hline \multicolumn{13}{|l|}{ Vitamins } \\
\hline Vitamin $A(\mu g R E)$ & 27 & $21 \cdot 8$ & 35 & $18 \cdot 4$ & EAR 500 & 30 & $32 \cdot 6$ & 34 & $19 \cdot 8$ & EAR 625 & 0.1454 & 0.0249 \\
\hline Vitamin $B_{1}(\mathrm{mg})$ & 25 & $20 \cdot 2$ & 26 & $13 \cdot 7$ & EAR 0.7 & 22 & 23.9 & 16 & $9 \cdot 3$ & EAR 0.9 & 0.6344 & 0.0064 \\
\hline Vitamin $B_{2}(\mathrm{mg})$ & 29 & 23.4 & 37 & 19.5 & EAR 1.1 & 29 & 31.5 & 27 & $15 \cdot 7$ & EAR 1.3 & 0.8757 & 0.0389 \\
\hline Vitamin $\mathrm{B}_{6}(\mathrm{mg})$ & 77 & $62 \cdot 1$ & 78 & $41 \cdot 1$ & EAR 1.3 & 51 & 55.4 & 79 & $45 \cdot 9$ & EAR 1.4 & 0.9113 & 0.0003 \\
\hline Folate $(\mu \mathrm{g})$ & 72 & $58 \cdot 1$ & 112 & 59 & EAR 320 & 53 & 57.6 & 74 & 43 & EAR 320 & 0.0122 & 0.1421 \\
\hline Vitamin $B_{12}(\mu \mathrm{g})$ & 43 & 34.7 & 57 & 30 & EAR $2 \cdot 0$ & 12 & 13 & 21 & $12 \cdot 2$ & EAR $2 \cdot 0$ & $<0.0001$ & 0.1446 \\
\hline Vitamin E (mg) & 71 & $57 \cdot 3$ & 80 & $42 \cdot 1$ & Al 7 & 67 & $72 \cdot 8$ & 96 & 55.8 & Al 10 & 0.001 & 0.0016 \\
\hline Vitamin C (mg) & 16 & $12 \cdot 9$ & 13 & 6.8 & EAR 30 & 11 & 12 & 15 & $8 \cdot 7$ & EAR 30 & 0.626 & 0.0068 \\
\hline Vitamin D $(\mu \mathrm{g})$ & 121 & 97.6 & 186 & 97.9 & Al 15.0 & 88 & $95 \cdot 7$ & 166 & 96.5 & Al 15.0 & 0.2813 & 0.9385 \\
\hline \multicolumn{13}{|l|}{ Minerals } \\
\hline $\mathrm{Ca}(\mathrm{mg})$ & 116 & 93.6 & 165 & $86 \cdot 8$ & EAR 1100 & 84 & 91.3 & 152 & 88.4 & EAR 1100 & 0.9384 & 0.0697 \\
\hline $\mathrm{Fe}(\mathrm{mg})$ & 14 & 11.3 & 15 & 7.9 & EAR 5 & 11 & 12 & 3 & 1.7 & EAR 6 & 0.1124 & 0.003 \\
\hline $\mathrm{Mg}(\mathrm{mg})$ & 99 & $79 \cdot 8$ & 120 & $63 \cdot 2$ & EAR 265 & 78 & $84 \cdot 8$ & 140 & 81.4 & EAR 350 & 0.0001 & 0.0031 \\
\hline $\mathrm{K}(\mathrm{mg}) \mathrm{Al}$ & 93 & 75 & 121 & $63 \cdot 7$ & Al 2800 & 83 & $90 \cdot 2$ & 147 & 85.5 & Al 3800 & $<0.0001$ & 0.0059 \\
\hline $\mathrm{Na}(\mathrm{mg})^{*}$ & 122 & 98.4 & 180 & 94.7 & Al $460-920$ & 91 & 98.9 & 172 & 100 & Al $460-920$ & 0.0242 & 0.4457 \\
\hline $\mathrm{Se}(\mu \mathrm{g})$ & 87 & $70 \cdot 2$ & 145 & $76 \cdot 3$ & EAR 50 & 65 & $70 \cdot 7$ & 127 & 73.8 & EAR 60 & 0.7243 & 0.8986 \\
\hline $\mathrm{Zn}(\mathrm{mg})$ & 50 & $40 \cdot 3$ & 67 & $35 \cdot 3$ & EAR 6.5 & 64 & $69 \cdot 6$ & 140 & 81.4 & EAR 12 & $<0.0001$ & 0.3353 \\
\hline \multicolumn{13}{|c|}{ Participants with El:BMR of between } \\
\hline \multirow{2}{*}{\multicolumn{13}{|c|}{$\begin{array}{l}0.9 \text { and } 2 \cdot 0 \\
\text { Vitamins }\end{array}$}} \\
\hline & & & & & & & & & & & & \\
\hline Vitamin $A(\mu \mathrm{g} R E)$ & 13 & $18 \cdot 1$ & 24 & $17 \cdot 8$ & EAR 500 & 10 & $22 \cdot 2$ & 22 & $17 \cdot 6$ & EAR 625 & 0.778 & 0.638 \\
\hline Vitamin $B_{1}(\mathrm{mg})$ & 10 & 13.9 & 13 & 9.6 & EAR 0.7 & 6 & 13.3 & 9 & $7 \cdot 2$ & EAR/RDI 0.9 & 0.557 & 0.124 \\
\hline Vitamin $B_{2}(\mathrm{mg})$ & 10 & 13.9 & 19 & $14 \cdot 1$ & EAR $1 \cdot 1$ & 9 & 20 & 21 & $16 \cdot 8$ & EAR 1.3 & 0.322 & 0.747 \\
\hline Vitamin $B_{6}(\mathrm{mg})$ & 42 & $58 \cdot 3$ & 48 & 35.6 & EAR 1.3 & 19 & $42 \cdot 2$ & 50 & 40 & EAR 1.4 & 0.760 & 0.004 \\
\hline Folate $(\mu \mathrm{g})$ & 36 & 50 & 79 & 58.5 & EAR 320 & 22 & 48.9 & 52 & 41.6 & EAR 320 & 0.020 & 0.723 \\
\hline Vitamin $B_{12}(\mu \mathrm{g})$ & 21 & $29 \cdot 2$ & 38 & $28 \cdot 2$ & EAR $2 \cdot 0$ & 2 & $4 \cdot 4$ & 14 & 11.2 & EAR $2 \cdot 0$ & $<0.001$ & 0.790 \\
\hline Vitamin E (mg) & 38 & $52 \cdot 8$ & 52 & 38.5 & Al 7 & 31 & 68.9 & 63 & $50 \cdot 4$ & Al 10 & 0.012 & 0.093 \\
\hline Vitamin C (mg) & 8 & $11 \cdot 1$ & 4 & 3 & EAR 30 & 1 & $2 \cdot 2$ & 10 & 8 & EAR 30 & 0.710 & 0.231 \\
\hline Vitamin D $(\mu \mathrm{g})$ & 69 & $95 \cdot 8$ & 133 & 98.5 & Al 15.0 & 44 & 97.8 & 119 & $95 \cdot 2$ & Al 15.0 & 0.338 & 0.580 \\
\hline \multicolumn{13}{|l|}{ Minerals } \\
\hline $\mathrm{Ca}(\mathrm{mg})$ & 65 & $90 \cdot 3$ & 120 & 88.9 & EAR 1100 & 42 & 93.3 & 113 & 90.4 & EAR 1100 & 0.524 & 0.597 \\
\hline $\mathrm{Fe}(\mathrm{mg})$ & 5 & 6.9 & 5 & 3.7 & EAR 5 & 2 & $4 \cdot 4$ & 0 & 0 & EAR 6 & 0.086 & 0.076 \\
\hline $\mathrm{Mg}(\mathrm{mg})$ & 53 & 73.6 & 88 & 65.2 & EAR 265 & 36 & 80 & 99 & $79 \cdot 2$ & EAR 350 & 0.0114 & 0.244 \\
\hline $\mathrm{K}(\mathrm{mg})$ & 50 & $69 \cdot 4$ & 88 & $65 \cdot 2$ & Al 2800 & 38 & 84.4 & 106 & 84.8 & Al 3800 & $<0.001$ & 0.399 \\
\hline $\mathrm{Na}(\mathrm{mg})^{\star}$ & 71 & 98.6 & 134 & 99.3 & Al $460-920$ & 45 & 100 & 125 & 100 & Al $460-920$ & 0.971 & 0.265 \\
\hline $\mathrm{Se}(\mu \mathrm{g})$ & 52 & $72 \cdot 2$ & 108 & 80 & EAR 50 & 31 & 68.9 & 88 & $70 \cdot 4$ & EAR 60 & 0.097 & 0.884 \\
\hline $\mathrm{Zn}(\mathrm{mg})$ & 21 & $29 \cdot 2$ & 42 & $31 \cdot 1$ & EAR 6.5 & 28 & $62 \cdot 2$ & 104 & $83 \cdot 2$ & EAR 12 & $<0.001$ & 0.354 \\
\hline
\end{tabular}

EAR, estimated average requirement; $\mathrm{Al}$, adequate intake.

* Al for all adult men and women. 
Table 7. Percentage of Mãori and non-Măori participants who did not meet the nutrient reference values for Australia and New Zealand ${ }^{(16)}$ for daily intake of micronutrients from food only by whether the participants used supplements or not

\begin{tabular}{|c|c|c|c|c|}
\hline & All & $\begin{array}{c}\text { No supplement } \\
\text { use }\end{array}$ & $\begin{array}{c}\text { Used } \\
\text { supplements }\end{array}$ & $P^{*}$ \\
\hline \multicolumn{5}{|l|}{ Māori } \\
\hline \multicolumn{5}{|l|}{ Vitamins } \\
\hline Vitamin A ( $\mu \mathrm{g} R E)$ & 24.4 & $35 \cdot 1$ & $12 \cdot 1$ & 0.0004 \\
\hline Vitamin $B_{1}(\mathrm{mg})$ & $20 \cdot 6$ & 23.7 & $16 \cdot 9$ & 0.259 \\
\hline Vitamin $B_{2}(\mathrm{mg})$ & $24 \cdot 4$ & 29.9 & $18 \cdot 1$ & 0.067 \\
\hline Vitamin $B_{6}(\mathrm{mg})$ & 54.4 & $58 \cdot 8$ & 49.4 & 0.210 \\
\hline Folate $(\mu \mathrm{g})$ & $56 \cdot 7$ & 63.9 & $48 \cdot 2$ & 0.034 \\
\hline Vitamin $B_{12}(\mu \mathrm{g})$ & 23.9 & $26 \cdot 8$ & $20 \cdot 5$ & 0.323 \\
\hline Vitamin E & $61 \cdot 1$ & 63.9 & $57 \cdot 8$ & 0.405 \\
\hline Vitamin C (mg) & $11 \cdot 1$ & $13 \cdot 4$ & 8.4 & 0.292 \\
\hline Vitamin D $(\mu \mathrm{g})$ & $96 \cdot 1$ & $96 \cdot 9$ & $95 \cdot 2$ & 0.552 \\
\hline \multicolumn{5}{|l|}{ Minerals } \\
\hline $\mathrm{Ca}(\mathrm{mg})$ & $92 \cdot 2$ & 94.9 & $89 \cdot 2$ & 0.157 \\
\hline $\mathrm{Fe}(\mathrm{mg})$ & 8.9 & $12 \cdot 4$ & 4.8 & 0.077 \\
\hline $\mathrm{Mg}(\mathrm{mg})$ & 79.4 & $85 \cdot 6$ & $72 \cdot 3$ & 0.028 \\
\hline $\mathrm{K}(\mathrm{mg})$ & 79.4 & 83.5 & 74.7 & 0.146 \\
\hline Se $(\mu \mathrm{g})$ & 68.9 & $70 \cdot 1$ & 67.5 & 0.704 \\
\hline $\mathrm{Zn}(\mathrm{mg})$ & 51.7 & $56 \cdot 7$ & $45 \cdot 8$ & 0.145 \\
\hline \multicolumn{5}{|l|}{ Non-Māori } \\
\hline \multicolumn{5}{|l|}{ Vitamins } \\
\hline Vitamin A ( $\mu \mathrm{g} R E)$ & $19 \cdot 0$ & $20 \cdot 7$ & $17 \cdot 7$ & 0.489 \\
\hline Vitamin $B_{1}(\mathrm{mg})$ & 11.5 & $9 \cdot 7$ & $12 \cdot 8$ & 0.364 \\
\hline Vitamin $B_{2}(\mathrm{mg})$ & 17.5 & $15 \cdot 9$ & $18 \cdot 7$ & 0.490 \\
\hline Vitamin $B_{6}(\mathrm{mg})$ & 42.5 & $47 \cdot 6$ & 38.9 & 0.107 \\
\hline Folate $(\mu \mathrm{g})$ & 51.1 & $53 \cdot 8$ & $49 \cdot 3$ & 0.405 \\
\hline Vitamin $B_{12}(\mu \mathrm{g})$ & 21.6 & $13 \cdot 1$ & $27 \cdot 6$ & 0.001 \\
\hline Vitamin E (mg) & 48.9 & 52.4 & $46 \cdot 3$ & 0.262 \\
\hline Vitamin C (mg) & 7.5 & $10 \cdot 3$ & 5.4 & 0.085 \\
\hline Vitamin D $(\mu \mathrm{g})$ & $97 \cdot 1$ & $96 \cdot 6$ & 97.5 & 0.588 \\
\hline \multicolumn{5}{|l|}{ Minerals } \\
\hline $\mathrm{Ca}(\mathrm{mg})$ & 87.4 & 89.7 & $85 \cdot 7$ & 0.276 \\
\hline $\mathrm{Fe}(\mathrm{mg})$ & 4.9 & $4 \cdot 8$ & 4.9 & 0.967 \\
\hline $\mathrm{Mg}(\mathrm{mg})$ & 71.8 & $82 \cdot 1$ & 64.5 & 0.0003 \\
\hline $\mathrm{K}(\mathrm{mg})$ & 73.9 & $80 \cdot 0$ & 69.5 & 0.028 \\
\hline Se $(\mu \mathrm{g})$ & 74.7 & $76 \cdot 6$ & 73.4 & 0.505 \\
\hline $\mathrm{Zn}(\mathrm{mg})$ & 57.8 & $64 \cdot 8$ & $52 \cdot 7$ & 0.024 \\
\hline
\end{tabular}

* $P$ value calculated using the Cochrane-Mantel-Haenszel test for comparison of two groups on a dichotomous response. Eighty-three Maori took supplements and ninety-seven did not. 203 non-Maori took supplements and 145 did not.

women) were the two main food sources of Se for both sexes, followed by eggs for Māori women and poultry for Māori men. Beef, bread and milk were the three main food groups contributing to $\mathrm{Zn}$ intake for Māori women and beef, bread and pork were the three main sources for Māori men.

\section{Food sources: non-Māori participants}

Vegetables were the main food sources of vitamin A for men (35\%) and women (36\%) followed by butter and margarine (men 13\%; women 12\%). Cereals, bread and vegetables were the three main food sources of folate intake for men. Contributions from these three food groups were in reverse order for women with milk also providing $10 \%$ of folate. The main food groups of vitamin $\mathrm{B}_{12}$ were milk (23\%), beef and veal (21\%), and fish and seafood (12\%) for men, contributing to more than half of their vitamin $\mathrm{B}_{12}$ intakes (55\%). Milk (24\%) and beef and veal (16\%) were also main food sources of vitamin $\mathrm{B}_{12}$ for women with other food group contributions more widely spread. Milk, butter/margarine and seafood were the three main food groups of vitamin D for men. Eggs and egg dishes replaced seafood in the top three food groups for women. Milk (34, 33\%) and cheese (11, 12\%) were the main contributors to dietary $\mathrm{Ca}$ for men and women. The main food sources contributing to Fe intake were cereals (20, 15\%) and bread (14, 12\%) for men and women, respectively. The beef and veal group was the third main contributor of Fe for men, while it was vegetables for women. For men, bread, cereals and milk were the main sources of $\mathrm{Mg}$, and bread, followed by fruit and cereals, were the main sources for women. The main food sources of $\mathrm{K}$ for men were potatoes, kumara and taro, vegetables and fruits, and for women the main source was fruits, followed closely by vegetables. The fish and seafood (17\%) group was the main contributor to Se for men, while the main source was bread (12\%) for women. Eggs and poultry were other important food sources. Beef and veal, bread and milk were the three main food groups contributing to $\mathrm{Zn}$ intake for both men and women.

\section{Discussion}

This is the first comprehensive assessment of micronutrient intake in Māori and non-Māori of advanced age. Overall, although most of the participants obtained an adequate EI, they met only some of the recommendations for micronutrient intake. As EI decreases with age ${ }^{(20)}$, obtaining adequate micronutrient intake presents a challenge for the oldest old, especially when recommendations are higher for older adults compared with younger age groups. Low intakes of folate, vitamin $\mathrm{E}, \mathrm{Ca}, \mathrm{Mg}, \mathrm{Se}$ and $\mathrm{Zn}$ were observed as reported in the diets of communityliving older adults ( $\geq 65$ years) in Western countries ${ }^{(33)}$.

Further, ethnic and sex differences in micronutrient intakes were evident. More than half of Māori participants did not meet the EAR for vitamin $\mathrm{B}_{6}$; more than half of Māori and non-Māori women did not meet the EAR for folate. More than half of all participants did not meet EAR for $\mathrm{Ca}, \mathrm{Mg}$ and Se, and more than half of men did not meet EAR for Zn. Similarly, the AI for vitamin $\mathrm{E}$ was not met by more than half of Māori participants. Seafood is a traditional food among Māori and was a main source of vitamin $\mathrm{B}_{12}$, vitamin $\mathrm{D}$ and Se. Colonial imposed restrictions on the gathering of seafood ${ }^{(34)}$ may impact on the ability to maintain a desirable diet for Māori. Efforts of Māori to negotiate acceptable self-determination have been fraught ${ }^{(35)}$ and those related to food gathering may contribute to inequities in health outcomes ${ }^{(36)}$. Micronutrient intake for Māori and non-Māori also differed by living situation, where lower intakes were observed among those who lived with others compared with those who lived alone or with a spouse. Māori had lower intakes of vitamin A, $\beta$-carotene, vitamin $\mathrm{B}_{6}$, folate, vitamin $\mathrm{C}$, and both Māori and non-Māori had lower intakes of $\mathrm{Mg}$ and $\mathrm{K}$. Participants who lived with others may have had a higher degree of dependency than those who lived alone or with a spouse. Their lower micronutrient intakes may reflect higher nutritional risk commonly observed in the dependent elderly ${ }^{(37)}$. A deeper understanding of the nutrient density of food intake among this vulnerable group is required. 


\section{Comparison with other studies}

The NZANS surveyed a representative sample of New Zealanders in 2008/09 using the 24-h MPR method of dietary assessment. Data were aggregated for those over age 71 years ( 159 men and 123 women) and for Māori over age 51 years (thirty-one men and forty-seven women) ${ }^{(19)}$. Two studies provided FFQ data for comparison: a longitudinal study in Mosgiel, New Zealand, in 1988 for men and women aged 65 and over ${ }^{(38)}$; and an Australian longitudinal study of 911 free-living older women aged 70-85 years $^{(7)}$. The National Health and Nutrition

Food groups contributing to $\geq 75 \%$ of vitamin A intake

$$
\text { Fruit } \text { III:1 }^{1: 1}
$$

Eggs and egg îin

dishes

Cheese III"

Dairy products inim

Milk inin

Butter and ifímilim|"

margarine

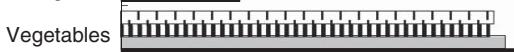

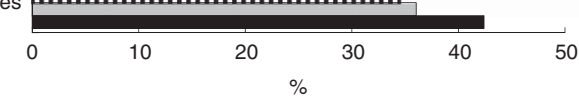

Food sources contributing to $\geq 75 \%$ of vitamin $B_{12}$ intake

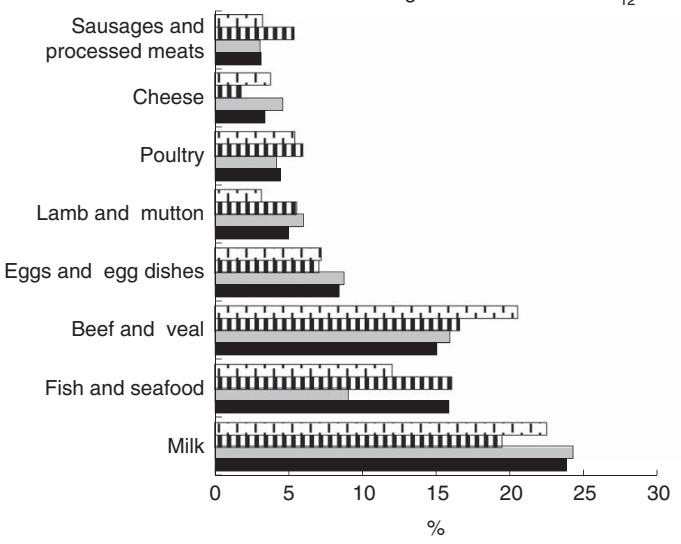

Food groups contributing to $\geq 75 \%$ of dietary Ca

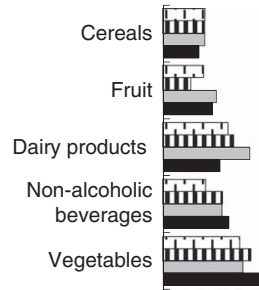

Cheese

Bread Inimini

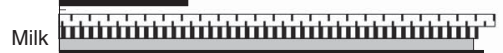
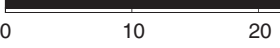

30
Examination Survey (NHANES) study in the USA provides specific details about intake and deficiency ${ }^{(39)}$ and European studies provide some comparative opportunities ${ }^{(20)}$. Overall, there are very little data on people in advanced age to provide adequate comparator data for LiLACS NZ. This highlights the importance of this study and other similar studies such as the Newcastle $85+$ study $^{(40)}$.

Low intake of vitamin $\mathrm{B}_{6}$ in Māori women participating in LiLACS NZ confirms NZANS (42\% low intake) and the Australian cohort ${ }^{(7)}$ findings. More than half of Māori women and all men in the current study appeared to consume less vitamin E
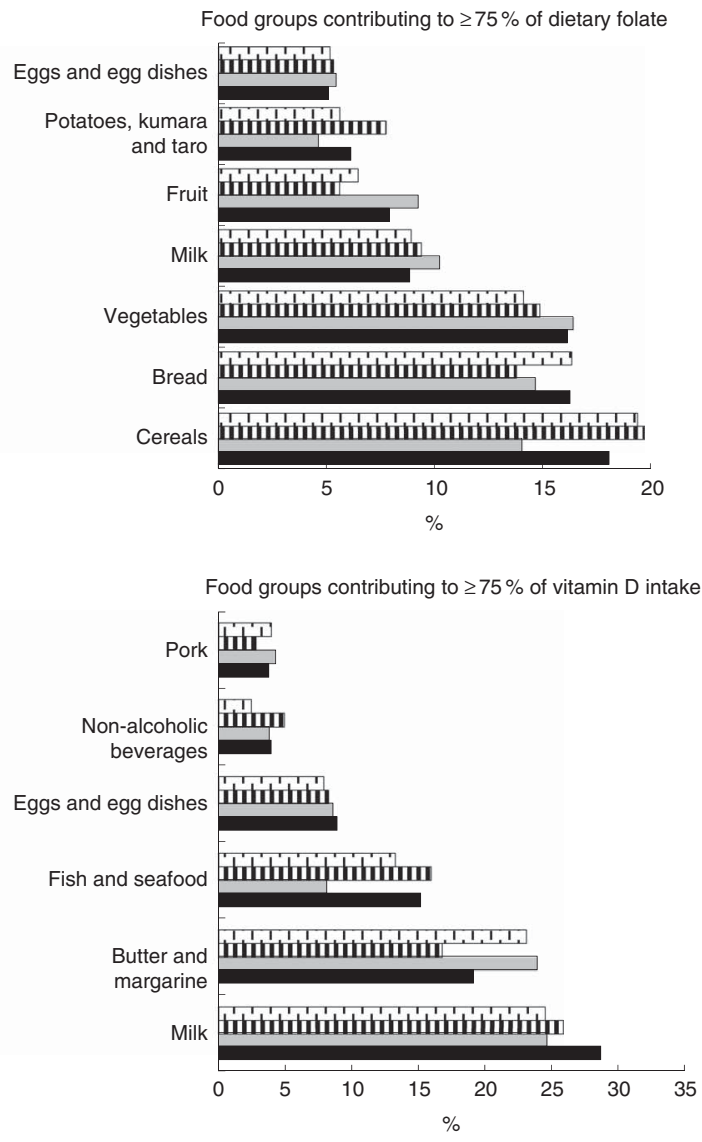

Food groups contributing to $\geq 75 \%$ of Fe intake

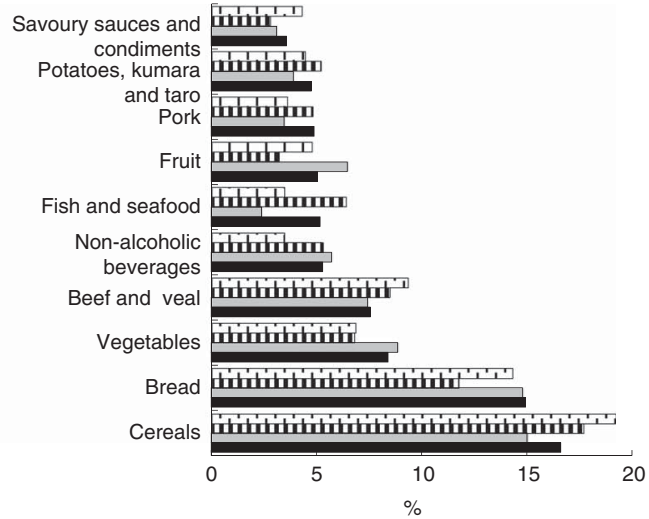

Fig. 1. (Continued on following page) 


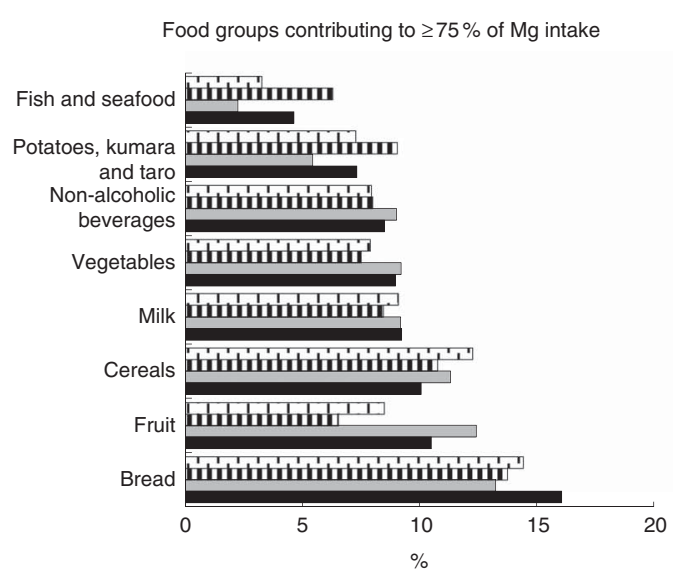

Food groups contributing to $\geq 75 \%$ of Se intake

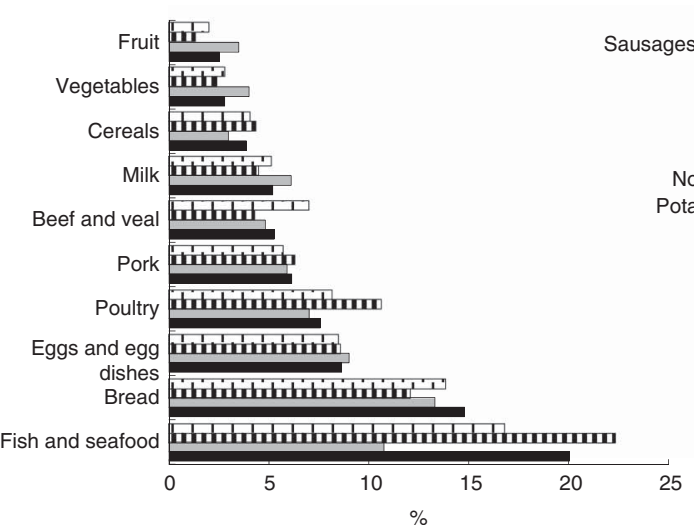

Food groups contributing to $\geq 75 \%$ of $\mathrm{K}$ intake

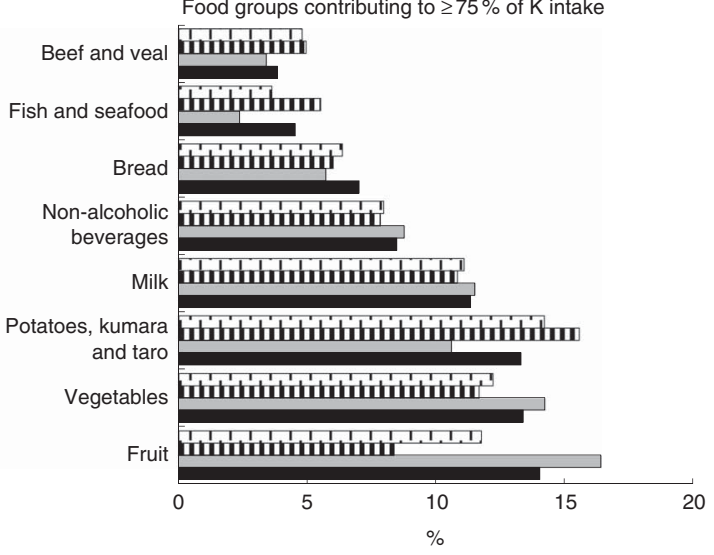

Food sources contributing to $\geq 75 \%$ of $\mathrm{Zn}$ intake

Pies and pasties thin

Plocessed meats

Eggs and egg dishes Eirit.

Fruit

Lamb and mutton

Grains and pasta

Non-alcoholic beverages Firing

otatoes, kumara and taro ing

Fish and seafood

Cheese Find

Poultry

Cereals

Vegetables minhind

Pork inimingmen

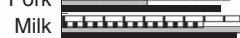

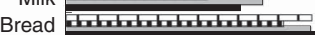

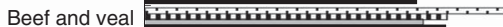

\begin{tabular}{llll}
\hline & 5 & 10 & 15
\end{tabular}

Fig. 1. (Continued from previous page) Percentage of food groups contributing to micronutrient intake by ethnic group and sex for vitamin $A$, folate, vitamin $B_{12}$, vitamin D, calcium, iron, magnesium, potassium, selenium, zinc. Milk: all milk (cow, soya, rice, goat and flavoured milk), milkshakes, milk powder. Dairy products: cream, sour cream, yogurt, dairy food, ice-cream, dairy-based dips. Cheese: Cheddar, Edam, specialty (Blue, Brie, Feta, etc.), Ricotta, cream cheese, cottage cheese, processed cheese. $\square$, Non-Māori men; $\square$, Māori men; $\square$, Non-Māori women; $\square$,Māori women.

than the recommended levels and less than older Australian women $^{(7)}$; a level of inadequacy was not found in the NZANS.

Dietary Ca has recently been emphasised in favour of supplementation because of an unexpected finding that $\mathrm{Ca}$ supplementation is associated with increased cardiovascular events in osteoporosis trials ${ }^{(41,42)}$. However, across most studies of older people, dietary $\mathrm{Ca}$ intake does not meet dietary recommendations ${ }^{(7,43,44)}$, as in LiLACS NZ; NZANS for women over 70 years $^{(19)}$, women in Mosgiel $^{(38)}$ and older women in Australia $^{(7)}$. Ca is not as well absorbed by the oldest age group $^{(16)}$ and thus suggestions that increased intake is needed seem reasonable. New ways of increasing dietary intake and intervention trials that study dose-response relationships to outcomes are needed, as not enough is known about Ca requirements during ageing ${ }^{(45)}$.

The EAR for Mg was not met by most LiLACS NZ participants. $\mathrm{Mg}$ requirements may change with age ${ }^{(46)}$ but clear conclusions are absent to set higher requirements ${ }^{(47)}$. Data from the NHANES III showed a progressive decrease in daily Mg intake with age ${ }^{(39)}$ with mean intakes for older men $(225 \mathrm{mg})$ and women (166 mg) being well below the RDA. A comprehensive review suggests that the dietary intake of $\mathrm{Mg}$ is inadequate in other elderly populations ${ }^{(48)}$, as was observed by Mosgiel ${ }^{(38)}$.
Although Mg is widely distributed in the food supply, it seems that older adults are less likely than younger adults to consume sufficient $\mathrm{Mg}$ to meet their needs ${ }^{(49)}$.

Se intakes were similarly marginal with over two-third of participants falling below the EAR, similar to the NZANS for those aged over 70 years. Se intake of the Mosgiel population was reported as adequate ${ }^{(38)}$; however, the level of intake was lower than that observed in LiLACS NZ participants. Whole population estimates from NHANES do not show similar low intakes $^{(50,51)}$ and Alaskans are not deficient in $\mathrm{Se}^{(52)}$. New Zealand soils are low in Se, and the New Zealand population's blood Se concentrations remain lower than those reported in other Western countries ${ }^{(53)}$. However, in the USA, frail older people have been found to be more likely to be deficient in Se than other population groups ${ }^{(54)}$. Intakes observed here need to be examined in relationship to serum levels and outcomes over time to fully understand the significance of low intake.

Most men did not meet the EAR for $\mathrm{Zn}$ intake, similar to the NZANS, where the highest prevalence of low intake for $\mathrm{Zn}$ across all age groups was for men aged $71+$ years $(90 \%)$ compared with only $28 \%$ of women. The Mosgiel study reported that $26 \%$ of men and $53 \%$ women had intakes below two-third of the RDA ${ }^{(38)}$. However, these findings should be 


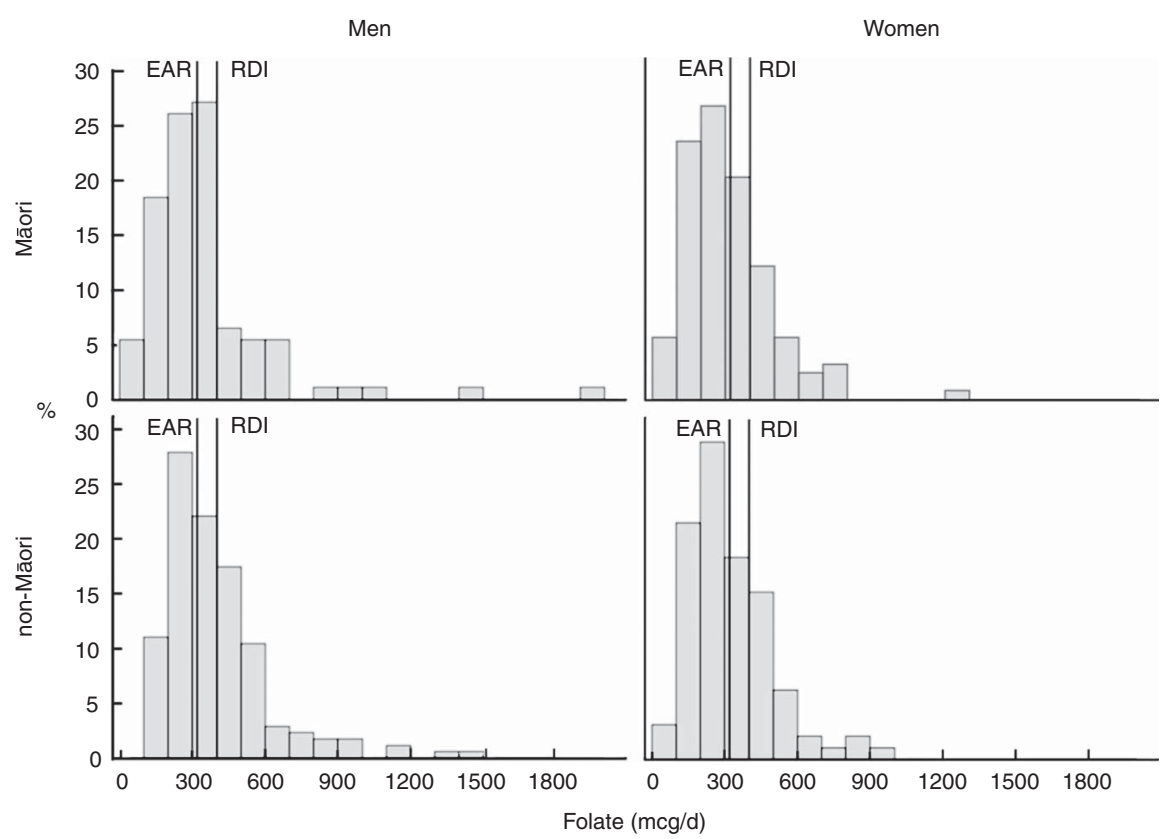

Fig 2. Intake distribution of folate with the estimated average requirement (EAR) and recommended daily intake (RDI) marked for Mãori and non-Mãori by sex.

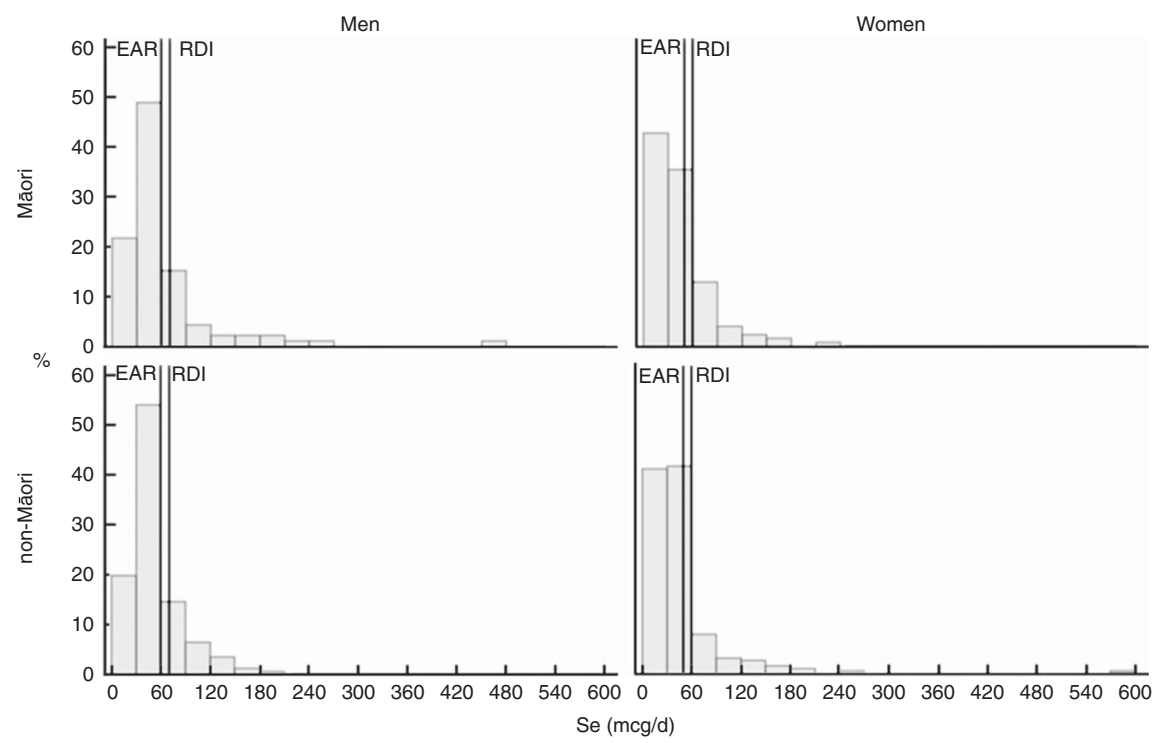

Fig 3. Intake distribution of selenium with the estimated average requirement (EAR) and recommended daily intake (RDI) marked for Māori and non-Māori by sex.

interpreted with caution, as the EAR for those aged over 70 years is based on experimental data from younger age groups and may be set too high for men.

\section{Micronutrient density}

Analysis by nutrient density showed that, although men in general consumed more energy than women, the micronutrient intake per MJ of energy was lower for men for several vitamins and minerals - for example, folate and Mg. Food group intake analysis showed that vegetables and fruits are more common sources of folate and $\mathrm{Mg}$ for women compared with cereals (folate) and potatoes, kumara and taro $(\mathrm{Mg})$ for men. Sex-related differences in food group contributions to Ca were not as apparent. The utility of using micronutrient density per MJ of EI, compared with absolute intakes, will be examined against longitudinal outcomes.

\section{Micronutrients of concern}

Vitamin $\mathrm{B}_{6}$ was lower in Māori women compared with other groups and may reflect their relatively lower EI compared with other subgroups of LiLACS NZ. As vitamin $\mathrm{B}_{6}$ is widely distributed in food, deficiency is rare, although it may be 
precipitated by anticonvulsant drugs that react with pyridoxal phosphate ${ }^{(55)}$. Dietary folate was low in women and may relate to their lower consumption of cereals and bread compared with men. However, fruit and vegetables were a common source of folate for women, and bioavailability of folates from these foods may be higher than that previously assumed ${ }^{(56)}$. Māori overall tended to have low intakes of vitamin $\mathrm{E}$ compared with non-Māori women, and this may reflect the consumption of smaller portions of plant foods high in fat, such as vegetable oils. $\mathrm{Ca}$ was the micronutrient with the highest prevalence of low intake in the diets of participants. Reduced supplies of $\mathrm{Ca}$ are associated with reduced bone mass and osteoporosis ${ }^{(57)}$, and a significant inverse association has been shown between total $\mathrm{Ca}$ intake and colorectal cancer ${ }^{(58)}$. Milk, cheese and bread were the main food sources of $\mathrm{Ca}$ in the current study with small contributions from other dairy products. As the optimal way to achieve adequate $\mathrm{Ca}$ intake is through diet, strategies are needed to increase milk and milk products intake in older people and to reduce high $\mathrm{Na}$ intakes, which may increase urinary $\mathrm{Ca}$ excretion. Both $\mathrm{Ca}$ and vitamin $\mathrm{D}$ are needed to ensure adequate absorption of $\mathrm{Ca}^{(59)}$. Vitamin D status in the New Zealand population is usually maintained by exposure to sunlight, and dietary sources of vitamin $\mathrm{D}$ are likely to make a relatively small contribution ${ }^{(60)}$. However, older people are at risk of insufficiency if they have limited sun exposure, are house bound or live in residential care. To ensure adequate vitamin D status of some older people, supplementation and food fortification may be needed. Targeting those at high risk of insufficiency and providing vitamin D supplementation will likely be a cost-effective approach.

The high prevalence of low Mg intake observed in LiLACS is potentially important and of concern because of its role in muscle function and age-related decline in physical performance $^{(61)}$. Low Mg intakes are also associated with lower bone mineral density in women ${ }^{(62)}$. Chronic $\mathrm{Mg}$ deficiency results in oxidative stress and low-grade inflammation ${ }^{(63)}$ and through activating the inflammatory process it is proposed to be involved in the ageing process itself ${ }^{(49)}$. Bread was the main food source of $\mathrm{Mg}$; further efforts may be needed to ensure older people have an AI of green vegetables, peas, beans, nuts and other sources of $\mathrm{Mg}$ such as fish and seafood.

Se has a vital role as an antioxidant in the regulation of the thyroid and immune function and may be important for maintenance of brain function ${ }^{(64)}$. For Māori, traditional foods such as seafood were the highest source of Se, which reinforces the importance of culturally significant foods. The inclusion of two Brazil nuts daily has been proposed as an effective way to improve Se status and negate the need for fortification or supplementation $^{(65)}$, and can be acceptable to older people, especially if ground and incorporated into other foods.

For $\mathrm{Zn}$, the highest prevalence of low intake was for non-Māori men. Expressed on a MJ food energy basis, Zn intake was $1.2 \mathrm{mg} / \mathrm{MJ}$ slightly lower compared with European men (aged $70+$ years) in the Zenith study ${ }^{(66)}$. Data are lacking on $\mathrm{Zn}$ status during normal ageing, and the implications of low intake are unknown; however, an $\mathrm{AI}$ of $\mathrm{Zn}$ is important for oxidative stress, immunity and cognitive functions ${ }^{(67)}$. We found that beef and veal, bread and milk were the main contributors to $\mathrm{Zn}$ intake, which is similar to findings for adults aged $71+$ years in the NZANS.

\section{Supplement usage}

Supplements users tended to have overall better dietary intakes, being more likely than non-users to meet micronutrient requirements from their diet alone. Māori men and women who took supplements were less likely to be deficient in their intake from food alone for vitamin $\mathrm{A}$, folate and $\mathrm{Mg}$, whereas nonMaori were less likely to be deficient in $\mathrm{Mg}, \mathrm{K}$ and $\mathrm{Zn}$, but more likely to be deficient in vitamin $\mathrm{B}_{12}$

In New Zealand, about a third of adults over 65 years have previously been reported to be regular dietary supplement users $^{(68)}$. Data from the USA suggest that more than half of older adults use dietary supplements ${ }^{(69)}$ and supplement use increases with age ${ }^{(70)}$. Supplement users are cited to be more likely to eat a balanced diet than non-users ${ }^{(70)}$, and this may reflect that taking supplements is part of an overall effort to improve health and wellness. Micronutrient requirements can, however, be achieved within a healthy well-balanced diet, which meets energy and macronutrient recommendations. Educational efforts to decrease the prevalence of micronutrient shortfalls need to focus on improving dietary intake and dietary variety and on improving the opportunity for older people to eat with others, which has been shown to improve dietary intake $^{(13)}$. Dietary supplement usage was lower among Māori than among non-Māori. This may reflect socio-economic disparities for Māori ${ }^{(71,72)}$ due to the high cost of dietary supplements. Further, disparities in dietary intake may contribute to inequity, which impacts health outcomes ${ }^{(72)}$.

\section{Limitations, strengths and weaknesses}

This study provides the first detailed examination of micronutrient intakes in Māori and non-Māori octogenarians. Dietary assessment has been conducted within a comprehensive longitudinal study, where attention to appropriate recruitment resulted in an acceptable engagement for both Māori and non-Māori participants. Data from this study add to the body of evidence to inform appropriate NRV in the very old.

The micronutrient reference values and recommendations for intakes for New Zealanders were developed on the basis of very low number of people in the older age groups or extrapolation from younger age groups and may bear no relationship to positive nutrition-related outcomes in older people. The micronutrient reference values and recommendations for intakes are presented as units of intake or a range of intakes regardless of body size or functional status factors, which are arguably more variable in older people than younger groups. Our study showed large variability in both absolute and energy-adjusted intakes. One main limitation of comparing absolute intakes to recommendations must be that interpretation is difficult for this age group where the variation in underlying functional and anthropometric status is so great and data on actual micronutrient requirements are lacking.

A further limitation is the lack of qualitative dialogue to accompany the quantitative data presented here, telling only a part of the story, particularly for Māori. Food is an important cultural activity for Māori. This design does not take into account the cultural value of food, the wairua, and spiritual 
significance of foods from a Māori perspective, which may equally impact nutrition-related outcomes.

Results need to be interpreted in light of considerations of potential inaccuracy in dietary recall related to cognitive decline in advanced age. We used the most acceptable and validated dietary assessment available ${ }^{(30)}$ and provided training, support and quality assurance. It is of concern that potential misreporting was more prevalent among Māori - a greater proportion of reports were outside the EI:BMR of between 0.9 and $2 \cdot 0$ - and this may have impacted the observed ethnic differences in dietary intake for some micronutrients (Table 6). However, the relevance and accuracy of the EI:BMR developed for younger age groups and applied to octogenarians in this study are not known. In further sensitivity analyses, we will examine differences in associations with outcomes.

\section{Conclusions}

A lack of evidence for age-specific NRV hinders the interpretation of nutrient adequacy; nonetheless, $\mathrm{Ca}, \mathrm{Mg}$, Se and folate (especially for women) are key micronutrients of concern in New Zealand. Milk and cheese provide important contributions to $\mathrm{Ca}$ intake, whereas bread is a key source of $\mathrm{Mg}$ and Se. Examination of dietary intake related to biochemical status and health outcomes will establish the utility of these observations.

\section{Acknowledgements}

Betty McPherson advised nutrition assessment for Māori and with Hone and Florence Kameta assisted with translation of the interview. The authors thank the organisations contracted to conduct the LiLACS NZ study in the communities of origin: Western Bay of Plenty PHO, Ngā Matāpuna Oranga Kaupapa Māori PHO, Rotorua Area Primary Health Services, Te Korowai Aroha Trust and Te Rūnunga o Ngati Pikiao, Te Rūnunga o Ngati Awa Research and Archives Trust, Te Rūnunga o Ngati Iripuaia and Te Whānau a Apanui Community Health Centre. The authors acknowledge the support of the Ministry of Health for manuscript production, and the authors thank all participants and their whānau for participation.

The Health Research Council of New Zealand programme grant (HRC 09/068B; main funding body) and Ngā Pae O te Māramatanga (the New Zealand National Centre for Research Excellence for Māori; funded Māori engagement and project management). A. A. is funded by the National Institute of Health Research UK as a Research Professor in translational research. The funders had no role in the design, analysis or writing of this article.

N. K. and A. A. conceived of the study and led its design; A. R. and M. M.-L. provided Māori leadership for the study; N. K., R. T., C. W. and K. H. were involved in formulating the research question; A. A. provided specialist training for MPR; K. H. provided project management oversight; C. W., R. T. and K. H. participated in data collection; S. A. M. and R. T. provided statistical analyses; C. W., R. T., N. K., A. A. and A. R. participated in manuscript preparation.

The authors declare that there are no conflicts of interest.

\section{References}

1. Statistics New Zealand (2013) Maori Population Estimates. 30 June 1991-2012. Wellington: Statistics New Zealand.

2. Ministry of Health (2011) Tatau Kura Tangata: Health of Older Mãori Chart Book. Wellington: Ministry of Health.

3. Statistics New Zealand (2007) New Zealand's 65+ Population' A Statistical Volume. Wellington: Statistics New Zealand.

4. Donini L, Savina C, Rosano A, et al. (2007) Systematic review of nutritional status evaluation and screening tools in the elderly. J Nutr Health Aging 11, 421-432.

5. Wham C, Teh R, Moyes S, et al. (2015) Health and social factors associated with nutrition risk: results from Life and Living in Advanced Age: A Cohort Study in New Zealand (LILACS NZ). J Nutr Health Aging 19, 637-645.

6. Roberts SB \& Rosenberg I (2006) Nutrition and aging: changes in the regulation of energy metabolism with aging. Physiol Rev 86, 651-667.

7. Zhu K, Devine A, Suleska A, et al. (2010) Adequacy and change in nutrient and food intakes with aging in a seven-year cohort study in elderly women. $J$ Nutr Health Aging 14, 723-729.

8. Drewnowski A \& Evans WJ (2001) Nutrition, physical activity, and quality of life in older adults: summary. J Gerontol A Biol Sci Med Sci 56, 89-94.

9. Genser D (2008) Food and drug interaction: consequences for the nutrition/health status. Ann Nutr Metab 52, Suppl. 1, 29-32.

10. Donini LM, Savina C \& Cannella C (2003) Eating habits and appetite control in the elderly: the anorexia of aging. Int Psychogeriatr 15, 73-87.

11. Elmadfa I \& Meyer AL (2008) Body composition, changing physiological functions and nutrient requirements of the elderly. Ann Nutr Metab 52, 2-5.

12. Morley JE (2001) Decreased food intake with aging. J Gerontol A Biol Sci Med Sci 56, 81-88.

13. Locher JL, Ritchie CS, Robinson CO, et al. (2008) A multidimensional approach to understanding under-eating in homebound older adults: the importance of social factors. Gerontologist 48, 223-234.

14. Heaney RP (2006) Barriers to optimizing vitamin $D_{3}$ intake for the elderly. J Nutr 136, 1123-1125.

15. Inzitari M, Doets E, Bartali B, et al. (2011) Nutrition in the agerelated disablement process. J Nutr Health Aging 15, 599-604.

16. National Health and Medical Research Council (2006) Nutrient Reference Values for Australia and New Zealand. Canberra: NHRMC.

17. National Health and Medical Research Council \& Ministry of Health (2005) Nutrient reference values for Australia and New Zealand. http://www.nrv.gov.au/nutrients/index.htm (accessed November 2012).

18. Wham C, Maxted E, Dyall L, et al. (2012) Korero te kai o te rangatira: nutritional wellbeing of Maori at the pinnacle of life. Nutr Diet 69, 213-216.

19. University of Otago \& Ministry of Health (2011) A Focus on Nutrition: Key Findings of the 2008/09 New Zealand Adult Nutrition Survey. Wellington: Ministry of Health.

20. de Groot CP, van den Broek T \& van Staveren W (1999) Energy intake and micronutrient intake in elderly Europeans: seeking the minimum requirement in the SENECA study. Age Ageing 28, 469-474.

21. Dyall L, Kepa M, Hayman K, et al. (2013) Engagement and recruitment of Māori and non-Māori people of advanced age to LiLACS NZ. Aust N Z J Public Health 37, 124-131.

22. Hayman K, Kerse N, Dyall L, et al. (2012) Life and Living in Advanced Age: A Cohort Study in New Zealand -Te Puawaitanga 
o Nga Tapuwae Kia Ora Tonu, LiLACS NZ: study protocol. $B M C$ Geriatr 12, 33.

23. Ministry of Social Development (2007) The Social Report 2007. Wellington: Ministry of Social Development.

24. Salmond C, Crampton P \& Atkinson J (2007) NZDep2006 Index of Deprivation. Wellington: Department of Public Health, University of Otago.

25. Salmond C, Crampton P \& Sutton F (1998) NZDep91: a New Zealand index of deprivation. Aust $N Z J$ Public Health 22 $835-837$.

26. University of Otago \& Ministry of Health (2011) Methodology Report for the 2008/09 New Zealand Adult Nutrition Survey. Wellington: Ministry of Health.

27. Hirani V \& Mindell J (2008) A comparison of measured height and demi-span equivalent height in the assessment of body mass index among people aged 65 years and over in England. Age Ageing 37, 311-317.

28. Fredrix EWHM, Soeters PB, Deerenberg IM, et al. (1990) Resting and sleeping energy expenditure in the elderly. Eur J Clin Nutr 44, 741-747.

29. Essink-Bot M-L, Krabbe PFM, Bonsel GJ, et al. (1997) An empirical comparison of four generic health status measures: the Nottingham Health Profile, the Medical Outcomes Study 36-Item Short-Form Health Survey, the COOP/WONCA charts, and the EuroQol instrument. Med Care 35, 522-537.

30. Adamson A, Collerton J, Davies K, et al. (2009) Nutrition in advanced age: dietary assessment in the Newcastle 85 plus; study. Eur J Clin Nutr 63, S6-S18.

31. The New Zealand Institute for Plant \& Food Research Limited (2011) New Zealand FOODfiles 2010 version 01. Palmerston North, New Zealand: The New Zealand Institute for Plant \& Food Research Limited and Ministry of Health.

32. Lökk J (2003) News and views on folate and elderly persons. J Gerontol A Biol Sci Med Sci 58, M354-M361.

33. ter Borg S, Verlaan S, Hemsworth J, et al. (2015) Micronutrient intakes and potential inadequacies of community-dwelling older adults: a systematic review. Br J Nutr 113, 1195-1206.

34. Lock K \& Leslie S (2007) New Zealand's Quota Management System: A History of the First 20 Years. Motu Working Paper 07-02. Wellington: Motu Economic and Public Policy Research.

35. Durie M (1998) Te Mana Te Kawanatanga: The Politics of Maori Self-Determination. Auckland: Oxford University Press.

36. Teh R, Kerse N, Kepa M, et al. (2014) Self-rated health, health related behaviours and medical conditions of Māori and nonMāori in advanced age: LiLACS NZ. N Z Med J 127, 13-29.

37. Fernández-Barrés S, Martín N, Canela T, et al. (2016) Dietary intake in the dependent elderly: evaluation of the risk of nutritional deficit. J Human Nutr Diet 29, 174-184.

38. Horwath CC, Campbell AJ \& Busby W (1992) Dietary survey of an elderly new Zealand population. Nutr Res 12, 441-453.

39. Ford ES \& Mokdad AH (2003) Dietary magnesium intake in a national sample of U.S. Adults. J Nutr 133, 2879-2882.

40. Davies K, Kingston A, Robinson L, et al. (2014) Improving retention of very old participants in longitudinal research: experiences from the newcastle 85+ study. PLOS ONE 9, e108370.

41. Grey A, Bolland M \& Reid R (2011) Calcium supplements and cardiovascular disease - picking the spin. Int J Clin Pract 65 , 226-227; author reply 227-228.

42. Grey A, Bolland M \& Reid IR (2009) Is calcium supplementation a risk factor for cardiovascular diseases in older women? Nutr Rev 67, 424; author reply 425.

43. Bailey RL, Dodd KW, Goldman JA, et al. (2010) Estimation of total usual calcium and vitamin D intakes in the United States. J Nutr 140, 817-822.
44. Ahmed M, Arcand J, Schermel A, et al. (2015) Analysis of the dietary intakes of canadian adults. FASEB J 29, 903.924.

45. Burckhardt P (2013) Calcium revisited: part I. BoneKEy Rep 2, 433.

46. Hunt CD \& Johnson LK (2006) Magnesium requirements: new estimations for men and women by cross-sectional statistical analyses of metabolic magnesium balance data. Am J Clin Nutr 84, 843-852.

47. European Food Safety Authority (EFSA) Panel on Dietetic Products, Nutrition and Allergies (NDA Panel) (2015) Scientific opinion on dietary reference values for magnesium. EFSA $J$ 13, 4186-4249.

48. Vaquero MP (2002) Magnesium and trace elements in the elderly: intake, status and recommendations. J Nutr Health Aging 6, 147-153.

49. Barbagallo M, Belvedere M \& Dominguez LJ (2009) Magnesium homeostasis and aging. Magnes Res 22, 235-246.

50. Fulgoni VL, 3rd, Keast DR, Bailey RL, et al. (2011) Foods, fortificants, and supplements: where do Americans get their nutrients? J Nutr 141, 1847-1854.

51. Nobmann ED, Ebbesson SO, White RG, et al. (1998) Dietary intakes among Siberian Yupiks of Alaska and implications for cardiovascular disease. Int J Circumpolar Health 57, 4-17.

52. Johnson JS, Nobmann ED, Asay E, et al. (2009) Dietary intake of Alaska Native people in two regions and implications for health: the Alaska Native Dietary and Subsistence Food Assessment Project. Int J Circumpolar Health 68, 109-122.

53. Thomson CD (2004) Selenium and iodine intakes and status in New Zealand and Australia. Br J Nutr 91, 661-672.

54. Smit E, Winters-Stone KM, Loprinzi PD, et al. (2013) Lower nutritional status and higher food insufficiency in frail older US adults. Br J Nutr 110, 172-178.

55. Clayton PT (2006) PT B6-responsive disorders: a model of vitamin dependency. I Inberit Metab Dis 29, 317-326.

56. Winkels RM, Brouwer IA, Siebelink E, et al. (2007) Bioavailability of food folates is $80 \%$ of that of folic acid. Am J Clin Nutr 85, 465-473.

57. Gennari C (2001) Calcium and vitamin D nutrition and bone disease of the elderly. Public Health Nutr $\mathbf{4}, 547-559$.

58. Chung M, Balk EM, Brendel M, et al. (2009) Vitamin D and calcium: a systematic review of health outcomes. Evid Rep Technol Assess (Full Rep) 183, 1-420.

59. Heaney RP (2008) Vitamin D and calcium interactions: functional outcomes. Am J Clin Nutr 88, 541S-544S.

60. Rockell JE, Skeaff CM, Williams SM, et al. (2006) Serum 25-hydroxyvitamin D concentrations of New Zealanders aged 15 years and older. Osteoporos Int 17, 1382-1389.

61. Veronese N, Berton L, Carraro S, et al. (2014) Effect of oral magnesium supplementation on physical performance in healthy elderly women involved in a weekly exercise program: a randomized controlled trial. Am J Clin Nutr 100, 974-981.

62. Orchard TS, Larson JC, Alghothani N, et al. (2014) Magnesium intake, bone mineral density, and fractures: results from the Women's Health Initiative Observational Study. Am J Clin Nutr 99, 926-933.

63. Barbagallo M \& Dominguez LJ (2010) Magnesium and aging. Curr Pharm Des 16, 832-839.

64. Zhang S, Rocourt C \& Cheng WH (2010) Selenoproteins and the aging brain. Mech Ageing Dev 131, 253-260.

65. Thomson CD, Chisholm A, McLachlan SK, et al. (2008) Brazil nuts: an effective way to improve selenium status. Am J Clin Nutr 87, 379-384.

66. Andriollo-Sanchez M, Hininger-Favier I, Meunier N, et al. (2005) Zinc intake and status in middle-aged and older European subjects: the ZENITH study. Eur J Clin Nutr 59, Suppl. 2, S37-S41. 
67. Prasad AS, Bao B, Beck FWJ, et al. (2004) Antioxidant effect of zinc in humans. Free Radical Biol Med 37, 1182-1190.

68. Ministry of Health (1999) NZ Food: NZ People. Key Results of the 1997 National Nutrition Survey. Wellington: Ministry of Health.

69. Qato DM, Alexander G, Conti RM, et al. (2008) Use of prescription and over-the-counter medications and dietary supplements among older adults in the United States. JAMA 300, 2867-2878
70. Dickinson A, Blatman J, El-Dash N, et al. (2014) Consumer usage and reasons for using dietary supplements: report of a series of surveys. J Am Coll Nutr 33, 176-182.

71. Chan WC, Wright C, Riddell T, et al. (2008) Ethnic and socioeconomic disparities in the prevalence of cardiovascular disease in New Zealand. $N Z$ Med J 121, 11-20.

72. Jatrana $S$ \& Blakely $\mathrm{T}$ (2008) Ethnic inequalities in mortality among the elderly in New Zealand. Aust $N Z J$ Public Health 32, 437-443. 